\title{
Vulnerability of ARID1A deficient cancer cells to pyrimidine synthesis blockade
} Zhigui $\mathrm{Li}^{1,4}$, Shijun $\mathrm{Mi}^{2,4}$, Oloruntoba I. Osagie ${ }^{1}$, Jing $\mathrm{Ji}^{1}$, Chia-Ping H. Yang ${ }^{2}$, Melissa Schwartz ${ }^{2}$, Pei Hui ${ }^{3}$ and Gloria S. Huang ${ }^{1 \star}$

\section{Affiliations}

${ }^{1}$ Department of Obstetrics, Gynecology \& Reproductive Sciences, Yale University, Yale School of Medicine, Yale Cancer Center, New Haven, Connecticut, 06520, USA.

${ }^{2}$ Department of Molecular Pharmacology, and Department of Obstetrics, Gynecology \& Women's Health, Albert Einstein College of Medicine, Albert Einstein Cancer Center, Bronx, New York, 10461, USA. ${ }^{3}$ Department of Pathology, Yale University, Yale School of Medicine, Yale Cancer Center, New Haven, Connecticut, 06520, USA. ${ }^{4}$ These authors contributed equally: Zhigui Li and Shijun Mi. ${ }^{\star}$ Correspondence and requests for materials should be addressed to G.S.H. (gloria.huang@yale.edu).

Running title: Targeting pyrimidine synthesis in ARID1A mutated cancer

Key words: Ovarian cancer; Pyrimidine synthesis; ARID1A

\section{Disclosure of Potential Conflicts of Interest}

G.S. Huang has received consulting fees/speaking honoraria from Bristol-Myers Squibb, Tesaro, and AstraZeneca Inc; these activities are unrelated to the work described in this manuscript. G.S. Huang is the inventor on a provisional patent filed by Yale University, related to the work described in this manuscript. No potential conflicts of interest were disclosed by the other authors. 


\section{ABSTRACT}

Here we report the discovery and preclinical validation of a novel precision medicine strategy for ARID1A-mutated cancer. Unbiased proteomics reveals for the first time that ARID1A protein (BAF250a) binds aspartate transcarbamoylase (ATCase), a key regulatory enzyme of the de novo pyrimidine synthesis pathway. Using isogenic paired ARID1A proficient/deficient cancer cell lines, we show that ARID1A protein deficiency (as occurs in ARID1A mutant cancers) leads to metabolic reprogramming and pyrimidine synthesis dependency. Pyrimidine synthesis blockade using the FDAapproved drug teriflunomide (a DHODH inhibitor) suppresses tumor growth and selectively induces DNA damage in ARID1A-deficient tumor models. Combining pyrimidine synthesis inhibition with DNA damage repair blockade, using teriflunomide and AZD6738 (an ATR inhibitor), achieves potent synergy and induces sustained tumor regression in $A R I D 1 A$-mutant ovarian cancer patient-derived xenografts (PDX). These pathway and DNA damage repair to induce regression in patient-derived xenograft

42 models of ARID1A-mutated cancer. 


\section{INTRODUCTION}

$A R I D 1 A$ is among the most commonly mutated tumor suppressor genes in human

cancer. The highest frequency of $A R I D 1 A$ mutations occur in gynecologic malignancies,

including clear cell ovarian carcinoma (46-57\%), endometrioid ovarian carcinoma

$(30 \%)$, uterine endometrial carcinoma (34\%) and uterine carcinosarcoma $(20-36 \%)^{1-3}$.

ARID1A mutations are also observed in $\sim 10-20 \%$ of diverse cancer types including gastric carcinoma, cholangiocarcinoma, bladder urothelial carcinoma, hepatocellular

carcinoma, esophageal adenocarcinoma, cutaneous melanoma, and colorectal

carcinoma. Mutations in ARID1A are strongly correlated with loss of protein

(mammalian SWI/SNF) chromatin remodeling complex, which modulates gene with transcription factors, coactivators, and corepressors ${ }^{5}$. Among BAF complex subunits, $A R I D 1 A$ is the most frequently mutated in human cancer. patients with $A R I D 1 A$ wildtype cancers. Overall survival is significantly shorter in patients with $A R I D 1 A$ mutated cancers compared to $A R I D 1 A$ wildtype cancers, when analyzing a pan-cancer cohort and when analyzing cancer-specific cohorts of patients with ovarian, hepatocellular, or pancreatic cancer ${ }^{6}$. Loss of ARID1A is also linked to shorter progression-free survival and chemoresistance ${ }^{7}$. The high frequency of $A R I D 1 A$ mutations in human cancer, and the unmet need for effective treatment for ARID1A deficient cancers, led us to undertake this study to uncover novel ARID1A functions associated with targetable therapeutic vulnerabilities. 
In this study, we used an unbiased proteomics approach to identify novel proteinprotein interactions of ARID1A. We show that ARID1A directly binds to ATCase, one of three key regulatory enzymes of the de novo pyrimidine synthesis pathway encoded by

72 the CAD gene (Carbamoyl-Phosphate Synthetase 2, Aspartate Transcarbamylase, And Dihydroorotase). ARID1A protein deficiency (as occurs in ARID1A-mutated cancers) leads to metabolic reprogramming characterized by an increased rate of de novo pyrimidine synthesis and selective vulnerability to pyrimidine synthesis blockade, thus identifying an Achilles' heel that can be therapeutically exploited. Furthermore, combination treatment with pyrimidine synthesis blockade and ataxia telangiectasia and rad3-related (ATR) kinase inhibition is potently synergistic and induces tumor regressions in vivo. Our results show a novel targetable function of ARID1A as a regulator of de novo pyrimidine synthesis and provide a therapeutic strategy to exploit the dependency of ARID1A deficient tumors on this metabolic pathway.

\section{RESULTS}

ARID1A interacts with the ATCase domain of CAD. To investigate unknown

functions of ARID1A, we used mass spectrometry to analyze the immunoaffinity-purified ARID1A complex with the aim to identify novel ARID1A-interacting proteins. First, the endogenous ARID1A complex was immunoprecipitated from ARID1A wildtype endometrial cancer cells $(\mathrm{KLE})^{8}$. The resulting Coomassie stained SDS-PAGE gel is shown in Fig. 1a. In addition to known BAF complex proteins, a $\sim 250 \mathrm{kD}$ protein band was observed and excised for analysis. Following trypsin proteolysis, the peptide 
MS/MS) and identified to be Carbamoyl-phosphate synthetase 2, Aspartate transcarbamoylase, and Dihydroorotase (CAD). Fragmentation spectra of CAD peptides is shown in Supplementary Fig. S1a. We also performed LC-MS/MS analysis of the immunopurified ARID1A complex from ARID1A wildtype ovarian cancer cells (ES2) ${ }^{9}$ and obtained similar results identifying CAD peptides. Based on functional similarities analysis (Supplementary Fig. S1b), CAD differs from known members of the ARID1A interactome (i.e. BAF complex proteins) and thus is newly identified to be an ARID1Ainteracting protein.

Immunoprecipitation using anti-ARID1A (Fig. 1b) and anti-CAD (Fig. 1c) antibodies followed by immunoblotting confirms the interaction between endogenous ARID1A and CAD in cell lines that express wild-type ARID1A. Co-immunoprecipitation results from ARID1A-wildtype ovarian cancer $(E S 2)^{8}$ cells are shown in Fig. 1 . Similar results are observed in ARID1A-wildtype endometrial cancer cells (KLE), data not shown. In addition to ARID1A, a core BAF complex subunit is SMARCA4 (also known as BRG1) ${ }^{10}$. As expected, SMARCA4 co-immunoprecipitates with ARID1A (Fig. 1b). In contrast, SMARCA4 does not co-immunoprecipitate with CAD, nor does CAD coimmunoprecipitate with SMARCA4 (Fig. 1c, 1d). ARID1A's interaction with CAD appears to be distinct from its known role as a BAF complex member. The nature of the protein-protein interaction of CAD and ARID1A was further investigated. Recombinant GST-tagged full-length CAD and CAD protein fragments, corresponding to the protein domains shown in Fig.1e, were expressed in Escherichia coli. The in vitro interaction of full-length CAD with ARID1A was demonstrated by GST pulldown assay (Fig. 1f). By using recombinant GST-tagged CAD fragments as bait, the 
ARID1A-interacting domain of CAD is localized to the aspartate transcarbamylase (ATCase) domain (Fig. 1f). In the next set of experiments, GST-ATCase was used as the bait, and full-length ARID1A, N-terminus ARID1A, or C-terminus ARID1A was expressed in HEK293 (Fig. 1g). GST-ATCase pulls down full-length ARID1A, as expected, and C-terminus ARID1A but not N-terminus ARID1A (Fig. 1h). Thus, ATCase is the ARID1A-interacting domain of CAD, and the C-terminus of ARID1A binds to ATCase.

ARID1A is a negative regulator of de novo pyrimidine biosynthesis. Next, we investigated the role of ARID1A as a potential regulator of CAD via its protein-protein interaction. ARID1A mutations in human cancers are typically associated with loss of ARID1A (BAF250A) protein expression due to truncating nonsense or frameshift mutations. To interrogate the functional consequences of ARID1A mutation and protein deficiency, we used previously validated short hairpin (sh) RNA vectors ${ }^{11}$ to knockdown $A R I D 1 A$, and expanded stably transfected clones, called shARID1A(a) and shARID1A(b), for further analysis. Knockdown of ARID1A was confirmed by immunoblotting (Fig. 2a and 2b). The resulting phenotype was evaluated relative to isogenic cells transfected with a non-targeting scrambled control shRNA (shCon) and untransfected cells.

As shown in Fig. 2a and 2b, ARID1A knockdown cells demonstrate higher total CAD levels relative to control cells. CAD activity is controlled via phosphorylation at key regulatory sites including serine 1859 . This CAD site is phosphorylated by ribosomal protein S6 kinase B1 (RPS6KB1, also known as S6K) ${ }^{12,13}$. Shown in Fig. 2a and 2b, serine 1859 phosphorylated CAD protein (P-CAD Ser1859) levels increase following 
ARID1A knockdown. In ARID1A knockdown cells, RPS6KB1 activity is similar as in control cells; evaluation of the RPS6KB1 canonical substrate, ribosomal protein S6 by immunoblotting is shown in Supplementary Fig. S2. Thus, total and phosphorylated CAD levels following ARID1A knockdown is not associated with altered RPS6KB1 activity and is inversely correlated with the ARID1A protein level.

The inverse correlation of ARID1A levels with CAD levels was confirmed by immunofluorescence analysis in several independent isogenic paired cell lines, and representative data is shown in Fig. 2c. Following ARID1A knockdown, the protein expression of phosphorylated CAD level is increase compared with control cells. Complementing these experiments, the effect of ARID1A restoration on CAD was determined using ARID1A-mutant ovarian cancer cell lines, SKOV3 and OVISE (Supplementary Table S10). Both of these cell lines contain ARID1A mutations, associated with loss of ARID1A protein expression. To restore ARID1A expression, SKOV3 cells and OVISE cells were stably transfected with a tetracycline inducible system to express full-length wildtype $A R I D 1 A$ and compared with empty vector control. Restored ARID1A protein expression was confirmed by immunoblotting (Fig. 2d). The total and phosphorylated CAD protein levels decrease in ARID1A restoration cells (Fig. $2 e$ and 2f). The inverse correlation of ARID1A and phosphorylated CAD levels in the ARID1A restoration cell lines is also evident by immunofluorescence (Fig. $2 \mathrm{~g}$ ).

Similarly, ARID1A restoration was done in HEC-1-A, an ARID1A-mutant endometrial cancer cell line. This cell line has two heterozygous truncating mutations at p.Q1835* and p.Q2115* associated with loss of ARID1A protein expression ${ }^{14}$. Following 
transfection with full-length ARID1A, compared with control empty vector, total and phosphorylated CAD levels decrease (Supplementary Fig. S3).

Collectively, these findings indicate that wildtype ARID1A functions as a negative regulator of $C A D$, wherein $A R I D 1 A$ deficiency results in an increase in total and phosphorylated CAD.

\section{ARID1A deficiency promotes de novo pyrimidine biosynthesis. The ATCase} enzymatic activity of CAD catalyzes the reaction of carbamoyl phosphate and aspartate to $\mathrm{N}$-carbamoyl aspartate, and is the first committed step in de novo pyrimidine biosynthesis ${ }^{15}$ (Fig. 3a). We next investigated whether ARID1A deficiency affects de novo pyrimidine synthesis. To quantify the rate of de novo pyrimidine synthesis, incorporation of ${ }^{14} \mathrm{C}$-radiolabelled aspartate into RNA and DNA was measured. RNA and DNA synthesized via the pyrimidine salvage pathway do not incorporate the ${ }^{14} \mathrm{C}$ radiolabelled aspartate, imparting specificity for measuring de novo pyrimidine synthesis flux. We found that $A R I D 1 A$ knockdown results in increased ${ }^{14} \mathrm{C}$ incorporation into RNA, indicating increased flux through the de novo pyrimidine synthesis pathway (Fig. 3b). Increased ${ }^{14} \mathrm{C}$ incorporation is similarly observed in DNA (data not shown). This demonstrates the inverse relationship of ARID1A levels and the rate of de novo pyrimidine synthesis.

An elevated rate of de novo pyrimidine biosynthesis may result in higher steady state levels of its product Uridine-5'-triphosphate (UTP). Thus, we examined UTP levels in ARID1A knockdown and restoration cell line panels. The UTP level is increased in ARID1A knockdown cells compared with control ARID1A wildtype cells (Fig. $3 \mathrm{c}$ and Supplementary Fig. S4a). The UTP level is reduced in ARID1A restoration cells 
183 compared with control ARID1A-mutant cells, SKOV3 and OVISE (Fig. 3d). Together,

184 these data indicate that the interaction of ARID1A and ATCase regulates de novo

185 pyrimidine biosynthesis flux and consequently, the pyrimidine nucleotide pool.

186

187

188

189

190

191

192

193

194

195

196

197

198

199

200

201

202

203

204

205

\section{ARID1A-deficient cells and tumors display sensitization to de novo pyrimidine} synthesis blockade therapy. We evaluated the effect of FDA-approved inhibitors of dihydroorotate dehydrogenase $(\mathrm{DHODH})$, the enzyme immediately downstream of CAD that catalyzes the conversion of dihydroorotate to orotate. As shown in Fig. $4 \mathrm{a}, A R I D 1 A$ knockdown cells are significantly more sensitive to the DHODH inhibitor teriflunomide. Similar results are observed with the DHODH inhibitor leflunomide (data not shown). ARID1A-mutant ovarian cancer cell lines SKOV3 and OVISE are sensitive to teriflunomide, while $A R I D 1 A$ restoration decreases sensitivity (Fig. $4 b$ and $4 c$ ).

Vulnerability of ARID1A deficient cells to de novo pyrimidine synthesis blockade was confirmed using isogenic ARID1A knockout and ARID1A wildtype HCT116 colorectal carcinoma cells. Homozygous deletion of ARID1A results from knock-in of premature stop codons (Q456*/Q456*). Compared to wildtype ARID1A cells, ARID1A knockout cells are hypersensitive to DHODH inhibitors (Supplementary Fig. S5).

In vivo validation is an important step in translation of scientific findings to clinical application. Therefore, we evaluated the therapeutic efficacy of DHODH inhibition using clear cell ovarian cancer xenografts (Fig. 4d). Xenograft-bearing mice were randomized to treatment with the DHODH inhibitor teriflunomide, or vehicle alone. Teriflunomide was administered intraperitoneally at a well-tolerated dosing regimen of $4 \mathrm{mg} / \mathrm{kg}$ every other day, corresponding to a human equivalent dose of $0.32 \mathrm{mg} / \mathrm{kg}$ every other day. This dose level is $\sim 30 \%$ lower than the FDA-approved dose level of $14 \mathrm{mg}$ daily used 
206 for treating multiple sclerosis ${ }^{16}$. As shown in Fig. 4d and 4e, teriflunomide selectively

207 suppresses tumor growth in ARID1A-deficient xenografts, compared to ARID1A-

208 wildtype xenografts (Supplementary Fig. S6). We also evaluated the effect of

209 teriflunomide in ARID1A-mutant SKOV3 tumor xenograft models (Fig. 4h).

210 Teriflunomide significantly improves animal survival compared to the vehicle treatment

211 group.

212 Patient-derived xenograft (PDX) models are particularly valuable for preclinical

213 validation. We used an ARID1A-mutant clear cell ovarian cancer PDX model (CTG-

214 2213; ARID1A truncating mutation at GIn211*) developed from direct implantation of

215 fresh viable human tumor tissue propagated in suitable mouse hosts. PDX models

216 accurately recapitulate tumor heterogeneity and predict clinical response to therapy.

217 Shown in Fig. 4i, tumor volume is significantly reduced in the teriflunomide treatment

218 group relative to the vehicle control group $(P<0.001)$. There was no weight loss or

219 toxicity observed in mice in either the treatment or vehicle control groups

220 (Supplementary Fig. S7). These data demonstrate the selective in vivo efficacy of

221 DHODH inhibition in multiple ARID1A-deficient cancer models.

222 DHODH inhibitor therapy induces DNA damage repair.

223 To investigate ARID1A-dependent differences in the cellular response to DHODH

224 inhibition, we evaluated DNA damage following drug treatment of isogenic ARID1A-

225 proficient and deficient cells. As shown by gamma-H2AX immunofluorescence and

226 immunoblotting (Fig. 5i-k), teriflunomide selectively induces DNA damage in ARID1A-

227 deficient cells relative to ARID1A-proficient ES2 cells. Teriflunomide treatment activates

228 the CHK1 DNA repair pathway, as shown by a robust increase in Ser-345 
229 phosphorylation of CHK1 in ES2-shARID1A cells compared with the ES2-shCon cells

230 (Fig. 5i-j). These results indicate that the cellular response of DHODH inhibition

231 depends on the ARID1A status. In ARID1A-deficient cells, induction of DNA damage by

232 teriflunomide triggers DNA damage repair signaling pathways that involve CHK1 kinase

233 activation.

234 ATR inhibition synergistically potentiates therapeutic effect of DHODH blockade

235 Since CHK1 kinase activation requires ATR activity, we hypothesized that ATR

236 inhibition would prevent activation of protective DNA repair signaling and thereby

237 enhance the efficacy of DHODH blockade.

reduced activity of alternate DNA repair pathways ${ }^{17}$. We confirmed enhanced sensitivity to ATR inhibition in ARID1A-knockout HCT116 colorectal carcinoma cells compared to ARID1A-wildtype HCT116 cells (Supplementary Fig. S9). We also evaluated the ATR inhibitor response in ARID1A-knockout ES2 ovarian carcinoma cells compared to ARID1A-wildtype ES2. We report in a separate manuscript the in vitro and in vivo evaluation of ATR inhibitors (e.g. AZD-6738, VX-970) in multiple ovarian and endometrial models. Our results in isogenic models demonstrate that ARID1A 246 deficiency confers sensitization to ATR inhibitors.

247 Next we evaluated the drug combination of teriflunomide with ATR inhibitors (AZD248 6738, VX-970). Drug combination analysis by the method of Chou and Talalay ${ }^{18}$ demonstrates synergy of concurrent teriflunomide and ATR inhibition in multiple 250 independent cancer cell lines (Fig. 5e-f; Supplementary Table S2-4). As shown using 251 knockdown and CRISPR knockout experiments, ARID1A-deficient cells are significantly 
252 more sensitive to combination therapy compared to isogenic ARID1A-proficient cells

253 (Fig. 5a-b; Supplementary Table S1, S5, S8; Supplementary Fig S10). ARID1A

254 restoration in ARID1A mutant cells results in drug antagonism and diminishes the

255 response to combination treatment (Fig. 5c-d, 5g-h; and Supplementary Table S6 and

256 S7). As predicted, combination treatment results in potent induction of DNA damage in

257 ARID1A-deficient cells compared to proficient cells (Fig. 5i-5k).

258 In vivo evaluation of Teriflunomide combined with ATR inhibitor AZD-6738 shows that

259 the combination treatment is highly efficacious (Fig. 5I-m and Supplementary Fig. S11).

260 Evaluated in two independent experiments using an aggressive ovarian cancer

261

262

263

264

265

266

267

268

269

270

271

272

273

274

xenograft model (ES2-shARID1A) and an ARID1A-mutant clear cell ovarian cancer

PDX model, the combination treatment is significantly more effective than single drug

treatments (Fig. 5l-m). The animals maintain normal activity and weight throughout drug

treatments which appear to be well tolerated. Sustained tumor regression is observed

following combination treatment in the ARID1A-mutant clear cell ovarian cancer PDXs

(Fig. 5m). Together, these data provide compelling preclinical data to support the

efficacy of this novel combination treatment for $A R I D 1 A$-mutant cancer.

\section{DISCUSSION}

Our results reveal a novel therapeutically targetable function of ARID1A as a regulator of de novo pyrimidine synthesis. We show for the first time that ARID1A deficiency results in vulnerability to pyrimidine synthesis blockade.

We found that pyrimidine synthesis blockade using currently available FDA-approved drugs such as teriflunomide selectively suppresses cellular proliferation and induces DNA damage in ARID1A-deficient cells. In vitro and in vivo experiments demonstrate 
275 that ARID1A deficiency predicts sensitivity to teriflunomide. Based on these data,

276 monotherapy with DHODH inhibitors may be useful for targeted treatment of cancers

277 with $A R I D 1 A$ mutations.

278 The antitumor efficacy of $\mathrm{DHODH}$ inhibition is enhanced by concurrently exploiting

279 the dependency of ARIDA-mutated cancers on ATR-mediated DNA repair. We show

280 that ATR inhibitors synergize with teriflunomide to potentiate DNA damage and

281 suppress cellular proliferation. This novel drug combination induced sustained tumor

282 regression in a highly aggressive tumor model, ARID1A-mutated ovarian clear cell

carcinoma PDXs. Combining pyrimidine synthesis blockade with DNA damage repair

inhibitors is an attractive strategy for clinical evaluation in biologically aggressive

ARID1A-mutated cancers.

We carried out protein interaction studies that show ARID1A directly binds to ATCase

287 (one of three enzymes encoded by the CAD gene). ATCase, the primary regulatory

thereby functioning as a cellular energy sensor input to this pathway. In addition to its enzymatic activity being positively regulated by a purine (ATP), ATCase is negatively regulated by a pyrimidine (CTP), enabling its critical function of maintaining nucleotide

292 pool balance between purines and pyrimidines ${ }^{19,20}$. Nucleotide pool balance is a key 293 determinant of DNA replication fidelity. Thus, regulation of ATCase by ARID1A could 294 play a role in maintaining nucleotide pool balance and DNA replication fidelity, and may contribute to ARID1A's tumor suppressor function.

In summary, we discovered that ARID1A regulates the de novo pyrimidine synthesis 297 pathway through an unexpected interaction with the energy-sensing enzyme ATCase. 
Metabolic reprogramming that results from ARID1A deficiency confers hypersensitivity to pyrimidine synthesis blockade, leading to therapeutic opportunities to repurpose FDA-approved inhibitors such as teriflunomide. Based on compelling data from in vitro and in vivo studies, we propose clinical trials of pyrimidine synthesis inhibitors alone and in combination with ATR inhibitors for precision therapy of ARID1A-mutated cancers.

\section{METHODS}

\section{Reagents}

\section{Plasmids}

HA-tagged full-length ARID1A was amplified by PCR from pCNA6-V5/His-ARID1A (provided by I.-M. Shih ${ }^{11}$ ) and subcloned into the pCIN4 expression vector ${ }^{21}$. To construct the expression plasmids for GST-CAD and GST-CAD fragments, CDNA sequences of full-length $C A D$ and its fragments were amplified by PCR from pcDNA3.1HisFlag-CAD (Addgene) and subcloned into the pGEX 4T-2 vector (GE Healthcare Life Sciences) for expression in BL21 bacteria. V5-tagged full-length BAF250a; V5-tagged BAF250a fragment, amino acids 1-1758; and V5-tagged BAF250a fragment, amino acids $1759-2285$, were created by G. R. Crabtree ${ }^{10}$ and obtained from Addgene. Short hairpin RNA (shRNA) lentiviral plasmids were kindly provided by I.-M. Shih ${ }^{8}$. The shRNA sequences for ARID1A are as follows: sh1(TRCN0000059090), target sequence CCTCTCTTATACACAGCAGAT, and sh2(TRCN0000059091), target sequence CCGTTGATGAACTCATTGGTT. The vector backbone is pLKO.1. V5/His-tagged pLenti-puro-LacZ and pLenti-puro-ARID1A were obtained from Addgene.

\section{Antibodies and Drugs}


321 Antibodies for ARID1A, CAD, CAD (IHC), and $\beta$-actin were from Bethyl Laboratories.

322 Antibodies for BRG1 (SMARCA4), Phospho-CAD (Serine 1859), Phospho-CHK1

323 (Serine 345), phosphor-Histone H2AX ( $\mathrm{HH} 2 \mathrm{AX}), \mathrm{HA}, \mathrm{V} 5, \mathrm{RPS6}$, Phospho-RPS6, and

324 GAPDH were from Cell Signaling Technology. Anti-lgG and HRP-labeled anti-rabbit

325 secondary antibodies were from Invitrogen. Leflunomide was from Enzo Life Sciences.

326 Teriflunomide and PF-4708671 were from Tocris. AZD6738 was from ChemScene.

327 VX970 (VE-822) was from Selleck Chemicals.

328 Cell Lines

329 Adherent cell lines were cultured in RPMI 1640 (Gibco) supplemented with 10\% fetal bovine serum (Gibco) and 1\% penicillin-streptomycin (Gibco) at $37^{\circ} \mathrm{C}, 5 \% \mathrm{CO}_{2}$. Low-

331 passage-number cells were used, and all cell lines tested negative for mycoplasma

332 using the MycoAlert Mycoplasma Detection Kit (Lonza). The following ovarian and

333 endometrial cancer cell lines were used: $\mathrm{ES}^{9}, \mathrm{KLE}^{8}, \mathrm{SKOV} 3, \mathrm{OVISE}$ (JCRB Cell

334 Bank), and HEC-1-A ${ }^{8}$ cells and their stably transfected subclones, as described below.

335 Cell lines were authenticated using the GenePrint10 kit (Promega) and matching to their 336 original profiles (ATCC). ARID1A protein expression status was confirmed by

337 immunoblotting. HEK293FT cells were from Thermo Fisher Scientific. ARID1A-knockout

338 HCT116 (homozygous truncating mutations, Q456*/Q456*) and ARID1A-wildtype

339 HCT116 colorectal carcinoma cells were from Horizon Discovery.

340 Mass Spectrometry Analysis of the Immunopurified ARID1A Complex

341 The immunoprecipitated ARID1A protein complex was separated by gel

342 electrophoresis, followed by peptide analysis of the digested gel bands by C18

343 reversed-phase chromatography using an UltiMate 3000 RSLCnano System (Thermo 
Scientific) equipped with an Acclaim PepMap C18 column (Thermo Scientific) and connected to a TriVersa NanoMate nanoelectrospray source (Advion) and a linear ion trap LTQ XL mass spectrometer (Thermo Scientific). Protein identification was performed using Mascot search engine v. 2.5.1 (Matrix Science) against the NCBI Homo sapiens database. Scaffold software v. 4.5.1 (Proteome Software) was used to validate the MS/MS peptide and protein identification based on $95 \%$ peptide and $99 \%$ protein probabilities, respectively.

\section{Functional similarities analysis}

Gene Ontology (GO) enrichment analysis by R packages was conducted as previously described ${ }^{22}$. Briefly, based on the semantic similarities of GO terms used for gene annotation, protein inside the interactome were ranked by the average functional similarities between the protein and its interaction partners. Functional similarity, which is defined as the geometric mean of their semantic similarities in molecular function (MF), cellular component (CC) and biological process(BP) aspect of GO, was designed for measuring the strength of the relationship between each protein and its partners by considering function and location of proteins. The distributions of functional similarities were demonstrated in supplementary Fig. S1. Proteins, which showed strong relationship in function and location among the proteins within the interactome, were essential for the interactome to exert their functions. The average of functional similarities was used to rank protein in the ARID1A interactome. A cutoff value of 0.5 was chosen. The source code is available upon request. Immunoblot Analysis and Co-Immunoprecipitation Assay 
For immunoblotting experiments, cells were lysed in BC200 lysis buffer $[20 \mathrm{mM}$ Tris-HCl (pH 7.5), $200 \mathrm{mM} \mathrm{NaCl}, 1 \mathrm{mM}$ EDTA, 0.2\% Nonidet P-40 (NP-40), freshly added complete protease inhibitor cocktail (Roche), and PhosSTOP phosphatase inhibitor (Roche)] or in boiling SDS lysis buffer [1\% SDS and $10 \mathrm{mM}$ Tris-Cl (pH 7.5)], as previously described ${ }^{23}$. Protein concentrations were quantified using a modified Lowry assay, and equal protein amounts were loaded onto a 10\% SDS-PAGE gel and separated by gel electrophoresis, followed by transfer to a nitrocellulose membrane.

The membrane was stained with Ponceau $S$ to confirm equal protein loading and then blocked in 2\% BSA in Tris-buffered saline with $0.1 \%$ Tween-20 (TBST). Primary antibody incubation was done for $2 \mathrm{~h}$ at room temperature or, for phospho-specific antibodies, overnight at $4^{\circ} \mathrm{C}$. An HRP-conjugated secondary antibody was used, followed by detection using enhanced chemiluminescence substrate (Pierce). Autoradiograph images were scanned and saved as unmodified Tiff images, and densitometry analysis was done with Image $(\mathrm{NIH})$ software.

For co-immunoprecipitation, cells were lysed in $\mathrm{BC} 150$ lysis buffer $[20 \mathrm{mM}$ Tris- $\mathrm{HCl}(\mathrm{pH}$ 7.5), $150 \mathrm{mM} \mathrm{NaCl}, 10 \%$ glycerol, $1 \mathrm{mM}$ EDTA, $0.2 \% \mathrm{NP}-40$, and freshly added complete protease inhibitor cocktail (Roche)]. Cell lysates were incubated with primary antibody or IgG control antibody at $4^{\circ} \mathrm{C}$ overnight, followed by incubation with Protein $\mathrm{A}$ Agarose beads (Sigma-Aldrich) for $1 \mathrm{~h}$ at $4^{\circ} \mathrm{C}$. After five washes with lysis buffer, the bound proteins were eluted from the beads in $2 x$ Laemmli SDS sample loading buffer at $95^{\circ} \mathrm{C}$ for $5 \mathrm{~min}$ and then loaded onto a $10 \%$ SDS-PAGE gel. Proteins were transferred to nitrocellulose membranes, and immunoblotting was performed as described above.

\section{Glutathione S Transferase (GST) Protein-Protein Interaction Assay}


Recombinant proteins, GST, GST-tagged CAD, and GST-tagged CAD fragments, shown in Fig. 2a, were purified as previously described ${ }^{24}$. HEK293T cells were transfected with pCIN4-HA-ARID1A using Lipofectamine 3000 (Invitrogen). Forty-eight hours later, the cells were lysed in lysis buffer [50 mM Tris-HCl (pH 8), 5 mM EDTA, 150 $\mathrm{mM} \mathrm{NaCl}, 0.5 \% \mathrm{NP}-40$, and freshly added $1 \mathrm{mM}$ DTT and complete protease inhibitor cocktail (Roche)]. The HEK293T lysates were then incubated for $2 \mathrm{~h}$ at $4^{\circ} \mathrm{C}$ with $25 \mu \mathrm{g}$ Glutathione Sepharose 4B beads (Amersham) bound to GST-CAD or its fragments. After washing in lysis buffer, bound proteins were eluted from the beads with $2 x$ Laemmli SDS sample loading buffer at $95^{\circ} \mathrm{C}$ for $5 \mathrm{~min}$, loaded onto a $10 \%$ SDS-PAGE gel, and then transferred to a nitrocellulose membrane and immunoblotted using the indicated primary antibodies.

To evaluate domain of ARID1A that potentially interacts with GST-ATCase, HEK293T cells were transfected with pCDNA6-V5/His.b (empty vector), pcDNA6-ARID1A 1-1758 (N-terminus), pcDNA6-ARID1A 1759-2285 (C-terminus), or pcDNA6-ARID1A (fulllength) expression plasmid using Lipofectamine 3000 (Invitrogen). Forty-eight hours later, the cells were lysed as described above. Lysates were then incubated for $2 \mathrm{~h}$ at $4^{\circ} \mathrm{C}$ with $25 \mu \mathrm{g}$ Glutathione Sepharose 4B beads (Amersham) bound to GST or GSTATCase. After washing in lysis buffer, bound proteins were eluted from the beads in $2 x$ Laemmli SDS sample loading buffer at $95^{\circ} \mathrm{C}$ for 5 min and then loaded onto an SDSPAGE gel, followed by transfer to a nitrocellulose membrane and immunoblotting using the indicated primary antibodies. 
412 shRNA lentiviral supernatants were produced using standard protocols, as previously

413 described ${ }^{11}$. Vectors were transfected into HEK293FT cells using X-tremeGENE 9 DNA

414 Transfection Reagent (Roche). Retroviral supernatants isolated at $48 \mathrm{~h}$ were diluted 1:1

415 in culture medium and used to infect the ARID1A-wildtype ES2 and KLE cell lines.

416 Stably transfected subclones were expanded using puromycin (Dot Scientific) drug

417 selection. For expression of ARID1A in the ARID1A-mutant HEC-1-A cell line, pCIN4-

418 HA-ARID1A was transfected using X-tremeGENE 9 DNA Transfection Reagent

419 (Roche), followed by selection with G418 (Gibco). For expression of ARID1A in the

ARID1A-mutant SKOV3 and OVISE cell lines, V5/His-tagged pLenti-puro-LacZ and

421 pLenti-puro-ARID1A were transfected using lentivirus. Lentivirus was produced using

422 HEK293FT cells with the second-generation packaging system pSPAX2 (Addgene

423 plasmid) and pMD2.G (Addgene plasmid). Stably transfected subclones were expanded

424 using puromycin and blasticidin (Gibco) drug selection.

\section{Targeted exon sequencing for ARID1A}

ARID1A mutations in SKOV3, A2780, HEC-1-A and OVISE cells were verified by

427 Sanger sequencing using a Applied Biosystems 3730xL DNA Analyzer (Thermo Fisher

428 Scientific, Inc.) and specific primers targeting exons 1, 2, 3, 18 and 20 (Supplementary

429 Table S9) of the ARID1A (ENST00000324856) CDS region were used according to

430 Jones, S. et al². Briefly, Genomic DNA was extracted using QIAamp UCP DNA Micro Kit

431 (Qiagen). PCR amplification with targeted primers was conducted using a touchdown

432 PCR protocol $\left(1\right.$ cycle of $96^{\circ} \mathrm{C}$ for $2 \mathrm{~min} ; 3$ cycles of $96^{\circ} \mathrm{C}$ for $10 \mathrm{sec}, 64^{\circ} \mathrm{C}$ for $10 \mathrm{sec}$,

$43370^{\circ} \mathrm{C}$ for $30 \mathrm{sec} ; 3$ cycles of $96^{\circ} \mathrm{C}$ for $10 \mathrm{sec}, 61^{\circ} \mathrm{C}$ for $10 \mathrm{sec}, 70^{\circ} \mathrm{C}$ for $30 \mathrm{sec} ; 3$ cycles

434 of $96^{\circ} \mathrm{C}$ for $10 \mathrm{sec}, 58^{\circ} \mathrm{C}$ for $10 \mathrm{sec}, 70^{\circ} \mathrm{C}$ for $30 \mathrm{sec} ; 41$ cycles of $96^{\circ} \mathrm{C}$ for $10 \mathrm{sec}, 57^{\circ} \mathrm{C}$ 
435 for $10 \mathrm{sec}, 70^{\circ} \mathrm{C}$ for $30 \mathrm{sec} ; 1$ cycle of $70^{\circ} \mathrm{C}$ for $\left.5 \mathrm{~min}\right)$. PCR products were purified

436 using QIAquick PCR Purification kit (Qiagen). PCR products were followed by Sanger

437 sequencing. Mutations in ARID1A in those verified cell lines by Sanger sequencing was

438 in Supplementary Table S10.

\section{Knockout of ARID1A in ES2 cell line}

440 The CRISPR-Cas9 system was used to according to Ran et a ${ }^{25}$. The CRISPR/Cas9

441 vector, pSpCas9(BB)-2A-Puro (PX459) V2.0 (ID: 62988), was obtained from Addgene

442 (MA, USA). The target site used in this study was 5'-

443 CACCGAGGGAAGCGCTGCTGGGAAT-3' that contains a part of the ARID1A

444 sequence. The PAM sequence is underlined. The target sequence was inserted into the

445 cloning site of the pSpCas9(BB)-2A-Puro (PX459) V2.0 vector. The cloned plasmid was

446 transfected into ES2 cells using Lipofectamine 3000. ES2 cells were selected in the

447 medium containing puromycin $(1 \mu \mathrm{g} / \mathrm{ml}) 72 \mathrm{~h}$ after transfection and screening for single

448 clones in about three weeks. ARID1A-null clones were identified by Western blotting

449 and confirmed by Sanger sequencing the targeted genome region by PCR amplification

450 with primers 5'-GTAAAACGACGGCCAGTTGCACGTTAGAGAACCACTCTG

$451-3$ ' and 5'-AACAGCTATGACCATGACAACCAGCAAAGTCCTCACC

$452-3^{\prime}$. 
454 Cells were plated in $60-\mathrm{mm}$ dishes $48 \mathrm{~h}$ prior to the experiment (cells grew to $85-90 \%$

455 confluent when the experiment started). Fresh medium was added to the subconfluent

456 cells, and $\left.5 \mu \mathrm{Ci} \mathrm{L-[U-}{ }^{14} \mathrm{C}\right]$ aspartic acid $(0.1 \mathrm{mCi} / \mathrm{mL}$, PerkinElmer $)$ was added to each

457 plate. After $6 \mathrm{~h}$ of incubation at $37^{\circ} \mathrm{C}$, the cells were lysed, and RNA and DNA were

458 prepared following the manufacturer's manual for the AllPrep DNA/RNA Mini Kit

459 (Qiagen). The amounts of RNA and DNA were quantified, and the radioactivity in each

sample was determined by liquid scintillation counting. $\left[{ }^{14} \mathrm{C}\right]$ Aspartate incorporation into

RNA or DNA was respectively normalized to the amount of RNA or DNA and expressed

464 UTP was examined with an enzyme immuno-based plate-reader assay according to the

466 in medium with or without drug treatment. For metabolite extraction, the medium was

467 aspirated, and the same number of cells were collected by trypsinizing and counting.

468 The cells were lysed by ultra-sonication (Qsonica Q125; Time: 2 min, Pulse: 15 s on/15 469 s off, Amplitude: 50\%). The insoluble material in lysates was pelleted by centrifugation

470 at $12,000 \mathrm{rpm}$ for $10 \mathrm{~min}$ at $4^{\circ} \mathrm{C}$. The metabolite-containing supernatants were

471 assessed using the UTP ELISA Kit. The plate was read at $450 \mathrm{~nm}$ with a standard

472 microplate reader. The UTP level was calculated using the formula $\left(\right.$ Relative $\left.O D_{450}\right)=$

$473($ Well OD 450$)-($ Mean Blank Well OD 450$)$

\section{Cytotoxicity assay}

475 Cells were seeded in 96-well plates, with 2000 cells in $100 \mu \mathrm{L} /$ well, and cultured for 24

476 h. The cells were treated with serial dilutions of the indicated drugs or without drug for 
477 an additional $72 \mathrm{~h}$. The cell number was determined using the sulforhodamine B (SRB)

478 assay, as previously described ${ }^{26}$. Briefly, cells were fixed with $20 \%$ trichloroacetic acid

479 (TCA), air-dried, and stained with 0.4\% SRB dissolved in $1 \%$ acetic acid. After washing, 480 protein-bound dye was solubilized with $10 \mathrm{mM}$ unbuffered Tris-base solution $(\mathrm{pH} 10.5)$

481 and detected at $510 \mathrm{~nm}$ using a microplate reader. Calculated the percentage of cell-

482 growth using the following formula:

$483 \%$ cell growth $=$ Absorbance sample/Absorbance untreated $\times 100$

484 Using CalcuSyn software (Biosoft), dose-effect curves were generated, and the drug

485 concentrations corresponding to a $50 \%$ decrease in cell number (IC50) were

486 determined.

\section{Immunofluorescence}

488 Cells plated on coverslips were kept in medium. For detecting proteins, coverslips were

489 fixed with $2 \%$ paraformaldehyde for 10 min, permeabilized with $0.5 \%$ Triton X-100 for 10 $490 \mathrm{~min}$, and blocked with 1\% BSA in $20 \mathrm{mmol} / \mathrm{L}$ Tris- $\mathrm{HCl}(\mathrm{pH} \mathrm{7.5)}$ for $20 \mathrm{~min}$. The

491 coverslips were then incubated with primary antibody (anti-ARID1A, 1:500; anti-CAD,

492 1:50; or P-CAD Ser1859, 1:50) overnight at $4^{\circ} \mathrm{C}$ and with secondary antibody for $2 \mathrm{~h}$ at

493 room temperature. For multicolor staining, an additional blocking step was conducted

494 after the first secondary antibody staining. DAPI was used to label the nucleus. Images

495 of cells were acquired using a BZ-X710 fluorescence microscope (KEYENCE) and

496 analyzed using ImageJ $(\mathrm{NIH})$.

\section{Immunohistochemistry (IHC)}

498 IHC was performed by incubating FFPE tissue section slides in antigen retrieval solution $499 \quad[0.01 \mathrm{M}$ sodium citrate $(\mathrm{pH} 6.0)]$ for $20 \mathrm{~min}$ in a pressure cooker. The slides were 
500 blocked in blocking solution (5\% goat serum and $2 \%$ BSA in TBS) for 30 min and then

501 incubated with primary antibodies (anti-ARID1A, 1:500; anti-CAD, 1:50) overnight at

$5024{ }^{\circ} \mathrm{C}$. The slides were then incubated with the secondary antibody from the EnVision GI2

503 Doublestain System (DAKO) for $10 \mathrm{~min}$ at room temperature, followed by DAB staining

504 for visualization. The slides were counterstained with hematoxylin and bluing in PBS,

505 dehydrated in graded alcohol, cleared in xylene, and coverslipped in Permount (Fisher

506 Scientific). Images were visualized using a BZ-X710 fluorescence microscope

507 (KEYENCE) and analyzed using ImageJ (NIH).

508 Animal model

509 Ethics statement

510 All animal procedures were conducted in accordance with a protocol approved by the

511 Institutional Animal Care and Use Committee at Yale University (Protocol number:

512 2017-20139).

513 Five mouse experiments were performed: (i) to assess the effect of teriflunomide on

514 ES2 cells in vivo, (ii) to assess the effect of teriflunomide on SKOV3 cells in vivo, (iii) to

515 assess the effect of teriflunomide on patient-derived xenograft model, (iv) to assess the

516 combination effect of teriflunomide and AZD6738 on ES2-shARID1A cells in vivo and

517 (v) to assess the combination effect of teriflunomide and AZD6738 on patient-derived

518 xenograft model. The i, ii, and iv experiments were experiments using subcutaneous

519 cell-xenograft models generated by injecting cells into the flanks of 6-week-old female

520 athymic NCr-nu/nu mice (Charles River Laboratories). The iii and v experiments were

521 performed using patient-derived xenografts (PDXs). Prkdc em26Cd52//2rgem26Cd22/NjuCrl

522 (NCG) mice were purchased from the Charles River. PDXs were generated by 
523 sectioning of Cryo ovarian tumor tissue (Champion Oncology) and engrafting tumor

524 chunks $(5 \times 5 \times 5 \mathrm{~mm})$ pieces subcutaneously to the 6 - to 8-week-old female mice.

525 Once the PDX tumor reached approximately $1,000 \mathrm{~mm}^{3}$, it was harvested and

526 transplanted for expansion in next generations, which were used for in vivo studies.

527 Animal-human dose translation was calculated as previously described ${ }^{27}$. Tumor

528 volumes were measured every other day by caliper to determine tumor volume using

529 the formula $\left(\right.$ length/2) $\times\left(\right.$ width $\left.^{2}\right)$. Animal weights were recorded, and mice were

530 observed for any toxicities. The experiment was terminated when the mean tumor

531 volume of the vehicle group reached $1000 \mathrm{~mm}^{3}$, and tumor xenografts were excised at

532 the time of euthanasia. Representative samples were flash-frozen in liquid nitrogen for

533 subsequent protein expression analysis by immunoblotting, as well as being formalin-

534 fixed paraffin-embedded (FFPE) for subsequent hematoxylin and eosin staining and

535 immunohistochemical analysis. FFPE sections were reviewed by the study pathologist

536 (P.H.), and cellular necrosis was quantified as \% cross-sectional area of the bisected

537 tumor xenografts.

538 (i) To assess the effect of teriflunomide on ES2 cells, xenograft models were generated

539 by injecting ES2-shCon or ES2-shARID1A cells $\left(1 \times 10^{6}\right.$ cells $)$ subcutaneously. When

540 the mean tumor volume reached approximately $100 \mathrm{~mm}^{3}$, the animals were randomized

541 into treatment groups; mice with xenograft volume $<20 \mathrm{~mm}^{3}$ or $>160 \mathrm{~mm}^{3}$ were

542 excluded. Teriflunomide was solubilized in DMSO and diluted to $0.5 \mathrm{mg} / \mathrm{mL}$ with PBS.

543 Mice were treated with teriflunomide $(4 \mathrm{mg} / \mathrm{kg})$ or vehicle intraperitoneally every other

544 day, as shown in Fig. 4d. 
545 (ii) To assess the effect of teriflunomide on SKOV3 cells, xenograft models were generated by injecting SKOV3 cells $\left[2 \times 10^{6}\right.$ cells mixed 1:1 (v/v) with Matrigel (BD

547 Biosciences)] subcutaneously. When the mean tumor volume reached approximately

$548100 \mathrm{~mm}^{3}$, the animals were randomized into treatment groups. Teriflunomide treatment was used the same way as in the ES2 xenograft models. The survival curve is shown in

550 Fig. 4h; the terminal tumor volume was $1000 \mathrm{~mm}^{3}$. (iii) To assess the effect of

551 teriflunomide on patient-derived xenografts (PDXs), the animals were randomized into

552 treatment groups once the mean tumor volume reached approximately $100 \mathrm{~mm}^{3}$.

553 Teriflunomide treatment was used the same way as in the ES2 xenograft models.

554 Tumor size was monitored every four days by a caliper, as shown in Fig. 4i. The

555 terminal tumor volume was $1000 \mathrm{~mm}^{3}$.

556 (iv) To assess the combination effect of teriflunomide and AZD6738 on ES2-shARID1A

557 cells in vivo, xenograft models were generated by injecting ES2-shARID1A cells [2 x $10^{6}$

558 cells mixed 1:1 (v/v) with Matrigel (BD Biosciences)] subcutaneously. Treatment with

559 was initiated $24 \mathrm{~h}$ after tumor injection, the animals were randomized into treatment

560 groups. Teriflunomide treatment was used the same way as in the ES2 xenograft

561 models. AZD6738 was solubilized in DMSO and diluted to $0.5 \mathrm{mg} / \mathrm{mL}$ with $10 \% 2$ -

562 hydroxypropyl-b-cyclodextrin. Treatment with AZD6738 (25 mg/kg) or vehicle was

563 performed daily by oral gavage, as shown in Fig. 51.

564 (v) To assess the combination effect of teriflunomide and AZD6738 on patient-derived 565 xenografts (PDXs), ARID1A deficient ovarian tumor tissues (Champion Oncology, CTG-

566 2213) were used in the study. The animals were randomized into treatment groups once 567 the mean tumor volume reached approximately $100 \mathrm{~mm}^{3}$. Teriflunomide and AZD6738 
568 treatment was used the same way as in the ES2 xenograft models. Tumor size was

569 monitored every four days by a caliper, as shown in Fig. $5 \mathrm{~m}$. The terminal tumor volume

570 was $500 \mathrm{~mm}^{3}$ in vehicle group.

571 Statistics

572 An independent-samples t-test was applied when two groups of data were compared.

573 Multiple-group comparisons were done using one-way analysis of variance (ANOVA)

574 with Tukey's post-test. Statistical analyses and graphing were performed using SPSS

57522 (IBM) and Prism 7 (GraphPad). P-values less than 0.05 were considered significant.

576 Acknowledgements

577 Funding for this research was provided by the Department of Defense Ovarian Cancer

578 Research Program Award W81XWH-16-1-0196 and the Reproductive Scientist

579 Development Program Seed Grant to G. S. Huang. We thank Dr. Jennifer T. Aguilan

580 and the comprehensive mass spectrometry services provided by the Proteomics Shared

581 Resource of the Albert Einstein College of Medicine, supported by the Cancer Center

582 Support Grant (NCI P30 CA013330). We thank the Shared Resources of the Yale

583 Cancer Center, supported by the Cancer Center Support Grant (NCI P30 P30

584 CA016359), and the YCC Scientific Publication Program supported by The Sands

585 Family Foundation. We thank Dr. le-Ming Shih for providing ARID1A vectors and short

586 hairpin RNA vectors used in this study. We acknowledge the Yale Genome Editing

587 Center for providing custom CRISPR/Cas constructs. We thank Dr. Susan Band Horwitz

588 for helpful discussions. 
G.S. Huang has received consulting fees/speaking honoraria from Bristol-Myers Squibb, Tesaro, and AstraZeneca Inc; these activities are unrelated to the work described in this manuscript. G.S. Huang is the inventor on a provisional patent filed by Yale University, related to work described in this manuscript. No potential conflicts of interest were disclosed by the other authors.

\section{Authors' Contributions}

Conception and design: G.S. Huang, Z. Li, S. Mi

Development of methodology: Z. Li, S. Mi, C-P.H. Yang, G.S. Huang Acquisition of data (provided animals, acquired and managed patients, provided facilities, etc.): Z. Li, S. Mi, O.I. Osagie, J. Ji, C-P.H. Yang, M. Schwartz, P. Hui, G.S. Huang

Analysis and interpretation of data (e.g., statistical analysis, biostatistics, computational analysis): Z. Li, S. Mi, O.I. Osagie, J. Ji, C-P.H. Yang, M. Schwartz, P. Hui, G.S. Huang

Writing, review, and/or revision of the manuscript: Z. Li, S. Mi, O.I. Osagie, J. Ji, CP.H. Yang, M. Schwartz, P. Hui, G.S. Huang

Study supervision: G.S. Huang

\section{Current Affiliations}

Shijun Mi, Laboratory of Personalized Genomic Medicine, Department of Pathology \& Cell Biology, Columbia University Medical Center, New York. Jing Ji, Department of Obstetrics \& Gynecology, the First Affiliated Hospital of Medical School, Xi'an Jiaotong 
614 University, Xi'an 710061, The People's Republic of China. Oloruntoba I. Osagie,

615 Queen's University Belfast, Northern Ireland, United Kingdom. Melissa Schwartz,

616 Department of Obstetrics, Gynecology and Women's Health, Division of Gynecologic

617 Oncology, Saint Louis University School of Medicine, St. Louis, Missouri.

618

619 Corresponding Author

620 Correspondence to G.S. Huang (gloria.huang@yale.edu).

621

622

623

624

625

626

627

628

629

630

631

632

633

634

635

636 
a

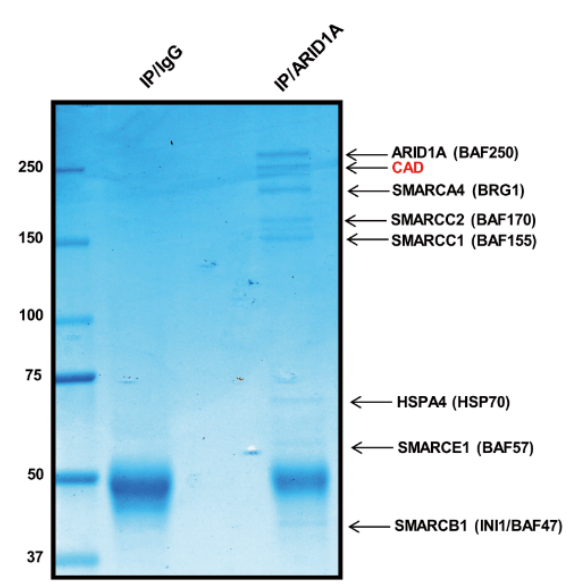

e

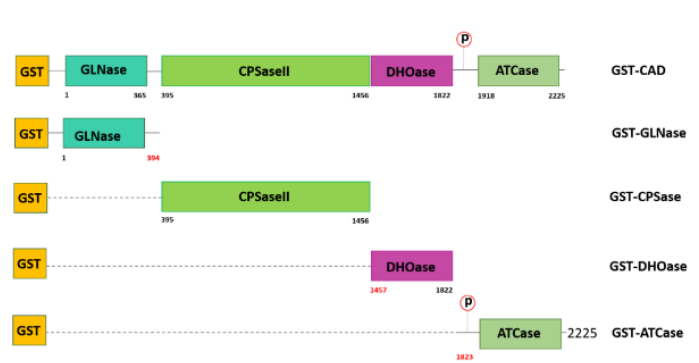

9

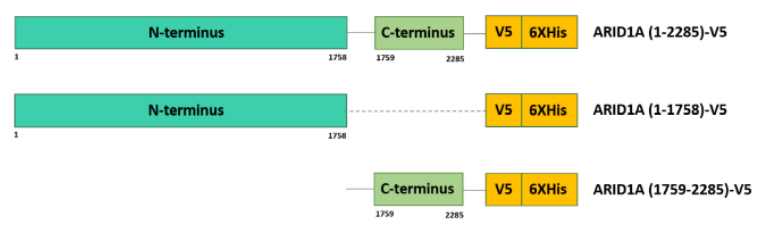

b

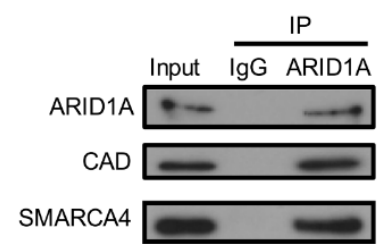

d Input $\frac{\mathrm{IP}}{\operatorname{IgG} \text { SMARCA4 }}$

ARID1A $\div \quad \cdot \rightarrow$

CAD $\square$
C

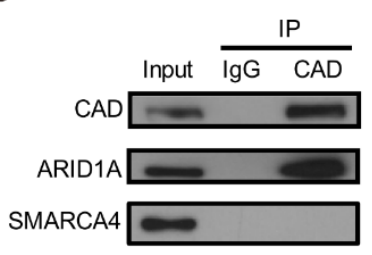

f
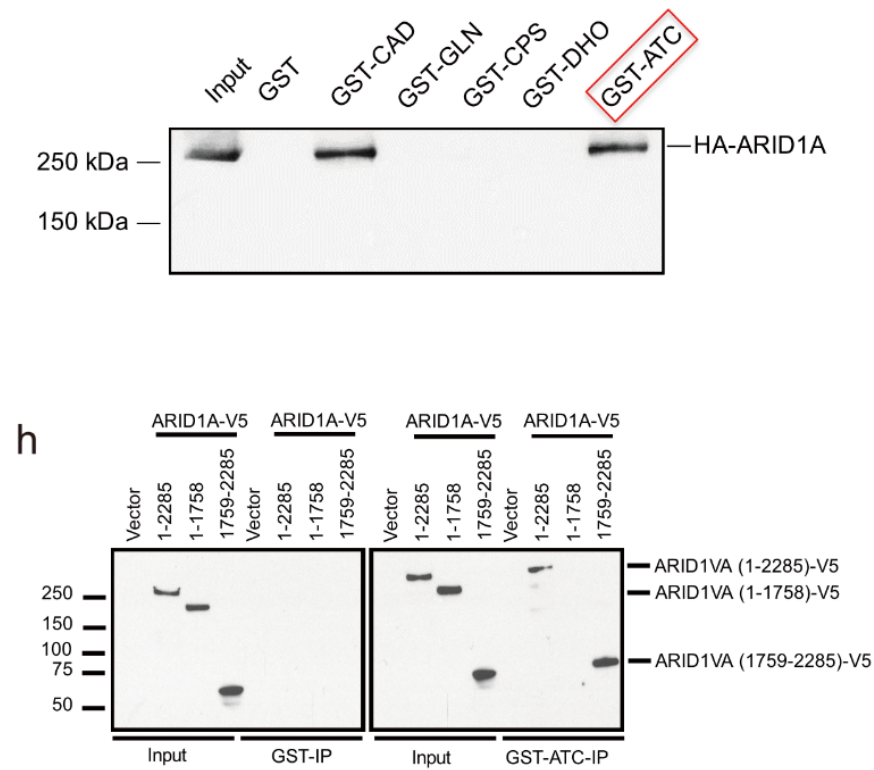

642 Figure 1. ARID1A interacts with CAD.

643 a, Coomassie blue staining of ARID1A complex immunopurified from an ARID1A644 wildtype cell line (KLE) is shown in the right lane, and immunopurified control IgG 645 complex is shown in the left lane. Arrows designate protein band identification by LC- 
MS/MS, with black text for ARID1A and SWI/SNF family members and red text for the multifunctional enzyme CAD.

b-d, Endogenous protein-protein interactions in an ARID1A-wildtype cell line (ES2) were assessed. Immunoprecipitation with the indicated antibody or control IgG antibody was performed, and immunoprecipitated proteins were detected by immunoblotting. At least two independent experiments were done, and representative immunoblots are shown. In (b), immunoprecipitation was done using an anti-ARID1A antibody. Both CAD and the core SWI/SNF subunit SMARCA4 co-immunoprecipitate with ARID1A. In (c), immunoprecipitation was done using an anti-CAD antibody. Endogenous ARID1A, but not SMARCA4, co-immunoprecipitates with CAD. In (d), immunoprecipitation was done using an anti-SMARCA4 antibody. Endogenous ARID1A, but not CAD, coimmunoprecipitates with SMARCA4.

\section{e, Recombinant, glutathione S-transferase (GST)-tagged proteins were made to} express full-length CAD or one of four unique non-overlapping CAD fragments. Each of the CAD fragments contains a functional enzyme component, as shown. CAD fusion proteins were expressed in bacteria.

f, Whole-cell lysates were prepared using HEK293T cells made to express HA-tagged full-length ARID1A. Shown are the results of a GST pulldown assay using recombinant GST-CAD fusion proteins (shown in A), followed by immunoblotting using an anti-HA antibody to detect HA-ARID1A. Recombinant full-length GST-CAD and the GSTATCase domain demonstrated in vitro binding to HA-tagged ARID1A.

\section{g, Recombinant ARID1A-V5 fusion proteins were made for expression of full-length} ARID1A (1-2285), N-terminal ARID1A (1-1758), or C-terminal ARID1A (1759-2285) in HEK293T cells.

h, The GST-ATCase fusion protein (but not the control GST protein assessed on the left side of the panel) demonstrated in vitro binding to V5-tagged, full-length ARID1A (12285) and to C-terminal ARID1A (1759-2285), but not to N-terminal ARID1A (1-1758). These data indicate that the protein-protein interaction of ARID1A and CAD is localized to the C-terminal regions of both CAD (ATCase domain, 1823-2225) and ARID1A (1759-2285). 
a
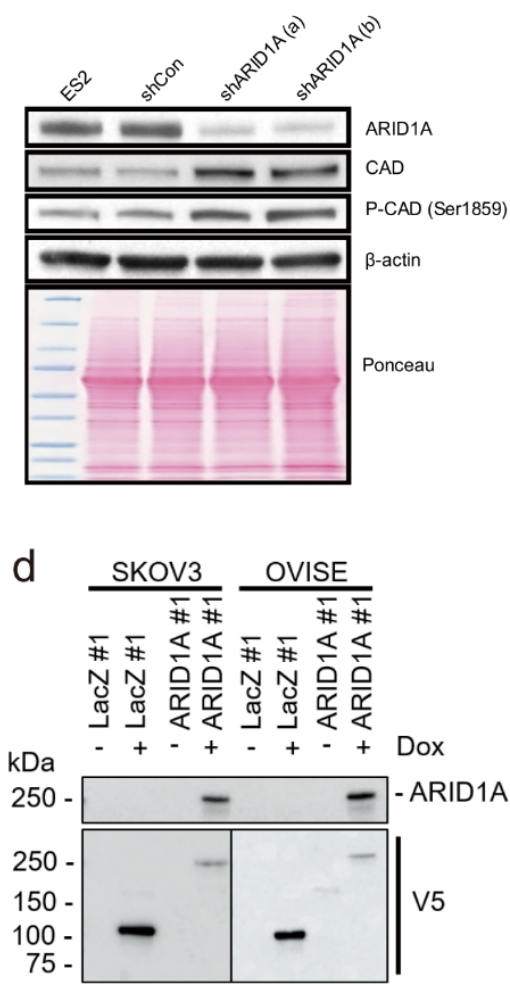

g

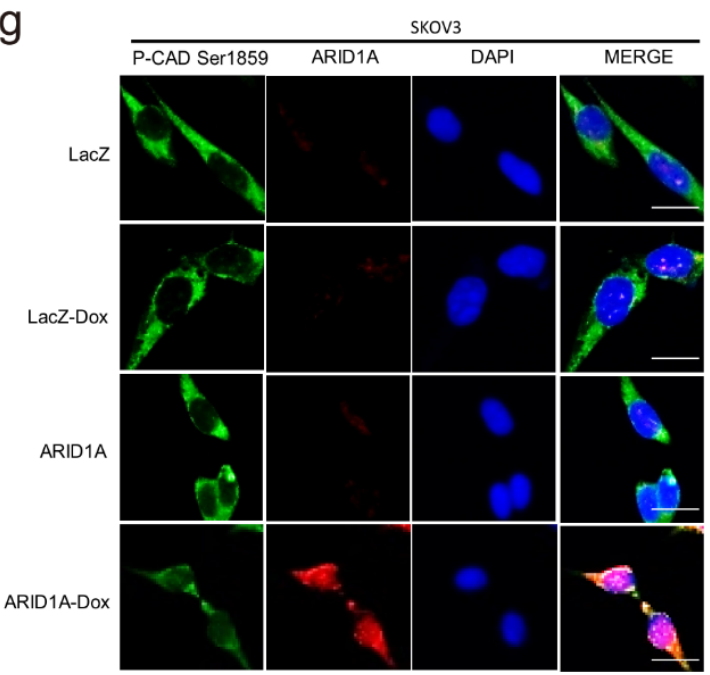

b

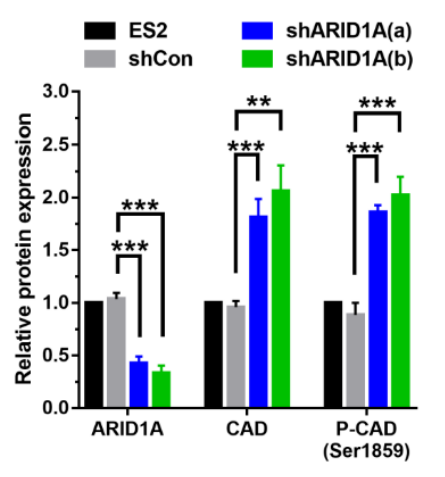

e

$\frac{\text { SKOV3 }}{\overline{\#}} \frac{\text { OVISE }}{\overline{\#}}$

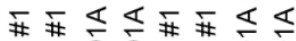

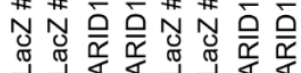
$\mathrm{kDa}-+\cdots+-+$ Dox 250 - $------D-C A D$ $250-\square-\cdots-\cdots---P-C A D$ 37 GAPDH
C

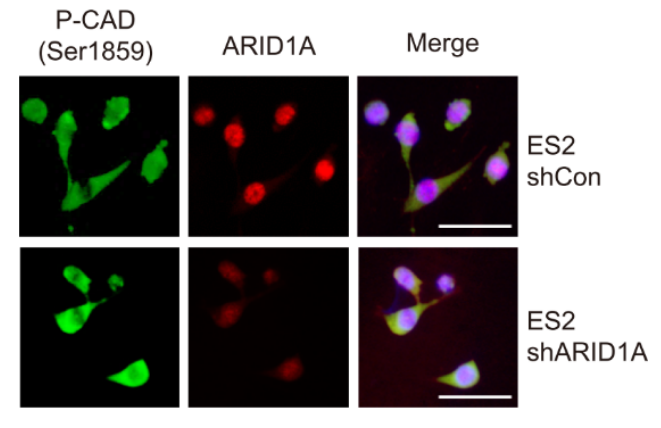

f $\mathbf{E}$ LacZ ARID1A

LacZ-Dox ARID1A-Dox

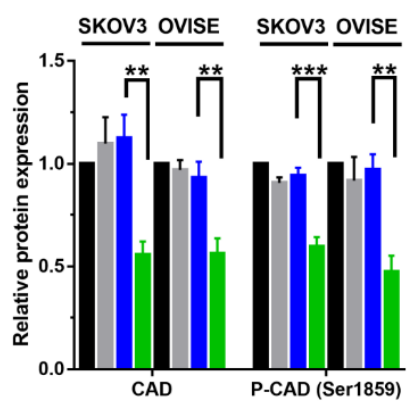

Figure 2. ARID1A is a negative regulator of CAD.

a, Immunoblotting using an anti-ARID1A antibody shows knockdown of ARID1A protein expression following stable transfection with short hairpin RNAs, shARID1A(a) and shARID1A(b), compared with control transfection with a non-targeting short hairpin RNA, shCon. Protein expression levels of total CAD and phosphorylated CAD increased in the ARID1A-knockdown cells compared to control cells, as shown by immunoblotting with anti-CAD and anti-phosphorylated-CAD antibodies. Equal protein loading is shown 
713

714

715

716

717

718

719

720

721

\section{6}

(7)

by immunoblotting with an anti- $\beta$-actin antibody and Ponceau staining of the nitrocellulose membrane. At least five independent experiments were done, and representative results are shown.

b, Quantitation of immunoblotting images in a by Image J. Data represent mean \pm s.e.m, $n=3$ independent experiments. One-way ANOVA with Tukey's post-test; ${ }^{* *} P<$ $0.01,{ }^{* * *} P<0.001$.

c, Representative immunofluorescence staining of ES2 cells for phosphorylated CAD Ser1859 (green) and ARID1A (red) is shown. P-CAD Ser1859 protein increased in the ARID1A-knockdown cells compared to control cells. Nuclei are indicated by DAPI staining. Scale bar, $40 \mu \mathrm{m}$.

d, Immunoblotting shows restoration of ARID1A protein expression following stable transfection with Lenti-puro-ARID1A-V5 (ARID1A) compared with control transfection with non-targeting Lenti-puro-LacZ-V5 (LacZ). Protein expression levels of ARID1A and V5 tag in the ARID1A-restoration cells compared to control cells were shown by immunoblotting with anti-ARID1A and anti-V5 antibodies. At least three independent experiments were done, and representative results are shown.

e, To evaluate the effect of ARID1A on CAD protein, immunoblotting using anti-CAD and anti-CAD Ser1859 antibodies was performed. CAD and phosphorylated CAD protein expression decreased following stable transfection with Lenti-puro-ARID1A (ARID1A) compared with control transfection with non-targeting Lenti-puro-LacZ (LacZ). Equal protein loading is shown by immunoblotting with an anti-GAPDH antibody. At least three independent experiments were done, and representative results are shown.

f, Quantitation of immunoblotting images in $\mathbf{d}$ by Image J. Data represent mean \pm s.e.m, $n=3$ independent experiments. One-way ANOVA with Tukey's post-test; ${ }^{* *} P<0.01$, ${ }^{* * *} P<0.001$.

g, Representative immunofluorescence staining of the SKOV3 (left) and OVISE (right) cell lines for phosphorylated CAD Ser1859 (green) and ARID1A (red) is shown. P-CAD Ser1859 protein decreased in the ARID1A-restoration cells following induction by doxycycline (Dox) for 2 days compared to control cells. Nuclei are indicated by DAPI staining. Scale bar, $20 \mu \mathrm{m}$.

19

20


a
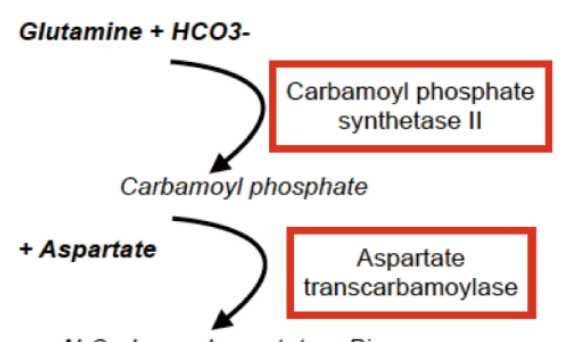

$N$-Carbamoylaspartate $+P i$

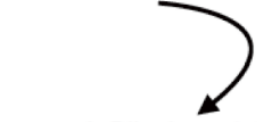

Dihydroorotase

L-Dihydroorotate $+\mathrm{H} 2 \mathrm{O}$

$+N A D$

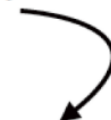

Dihydroorotate

dehydrogenase

Orotate + NAD+ $\mathrm{H}+$

+ PRPP

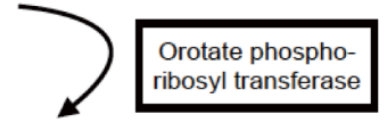

Orotidylate $+P P i$

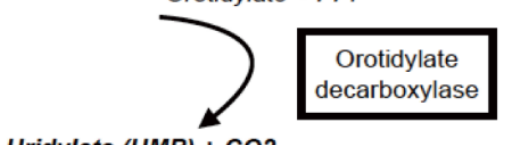

Uridylate $(U M P)+C O 2$ b

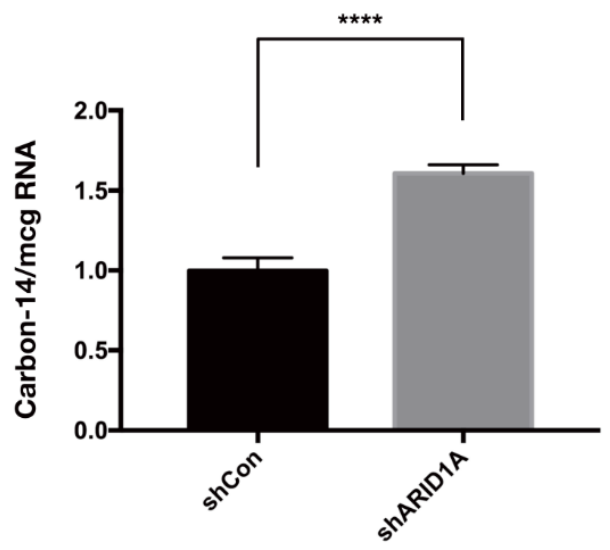

C

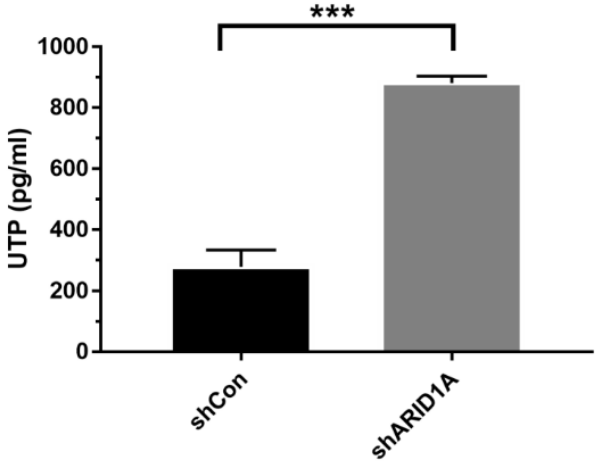

d

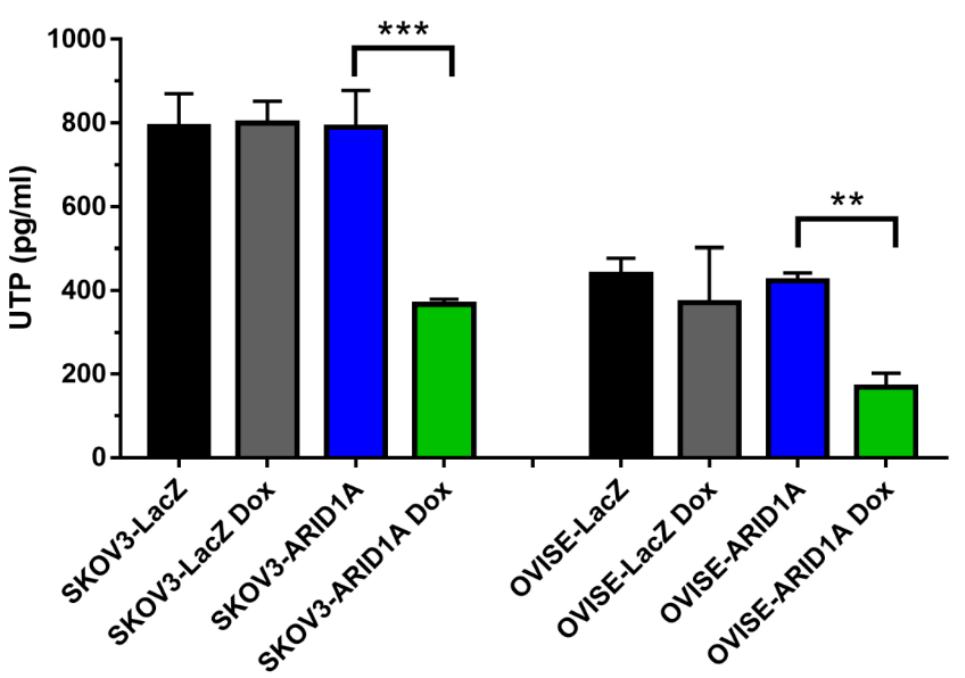

Figure 3. ARID1A deficiency promotes de novo pyrimidine synthesis.

727 a, The diagram shows the steps in the de novo pyrimidine synthesis pathway. Aspartate is incorporated by aspartate transcarbamoylase in the second step of de novo

729 pyrimidine synthesis.

b, To evaluate the effect of ARID1A knockdown on de novo pyrimidine synthesis, carbon-14-labeled aspartate was added to the growth media. After a 6-hour incubation, RNA was isolated from the cells, and carbon-14 incorporation into the RNA was 
746

747

748

749

750

751

752

753

754

755

756

757

758

759

760

761

762

763

764

765

766

767

768 quantified as the counts per minute (cpm) measured by a scintillation counter and was normalized to the amount of RNA (micrograms). The bar graph shows increased carbon-14 incorporation into RNA in ARID1A-knockdown cells relative to control cells. The mean \pm SD calculated from two independent experiments, each performed in two to four biological replicates, is shown. ${ }^{* * *} P<0.0001$, two-tailed $t$-test.

c, The cellular UTP level was evaluated in ARID1A-knockdown cells. UTP increased in ARID1A-knockdown ES2 cells relative to control cells transfected with a non-targeting short hairpin RNA, shCon. The UTP level in ES2 parental and another ARID1AKnockdown ES2 cells show similar result (Fig. S3a). Teriflunomide (Teri) treatment decreased UTP in the ES2 panel (Fig. S3b). Differences in UTP were evaluated using one-way ANOVA with Tukey's post-test; ${ }^{* *} P<0.001$.

d, UTP decreased in ARID1A-restoration cell lines (SKOV3, left, and OVISE, right) following induction by doxycycline (Dox) compared with control cells transfected with non-targeting Lenti-puro-LacZ (LacZ). Differences in UTP were evaluated using oneway ANOVA with Tukey's post-test; ${ }^{* *} P<0.01,{ }^{* * *} P<0.001$.

9

0

1

2

3

5

56

58

59

61

62

63



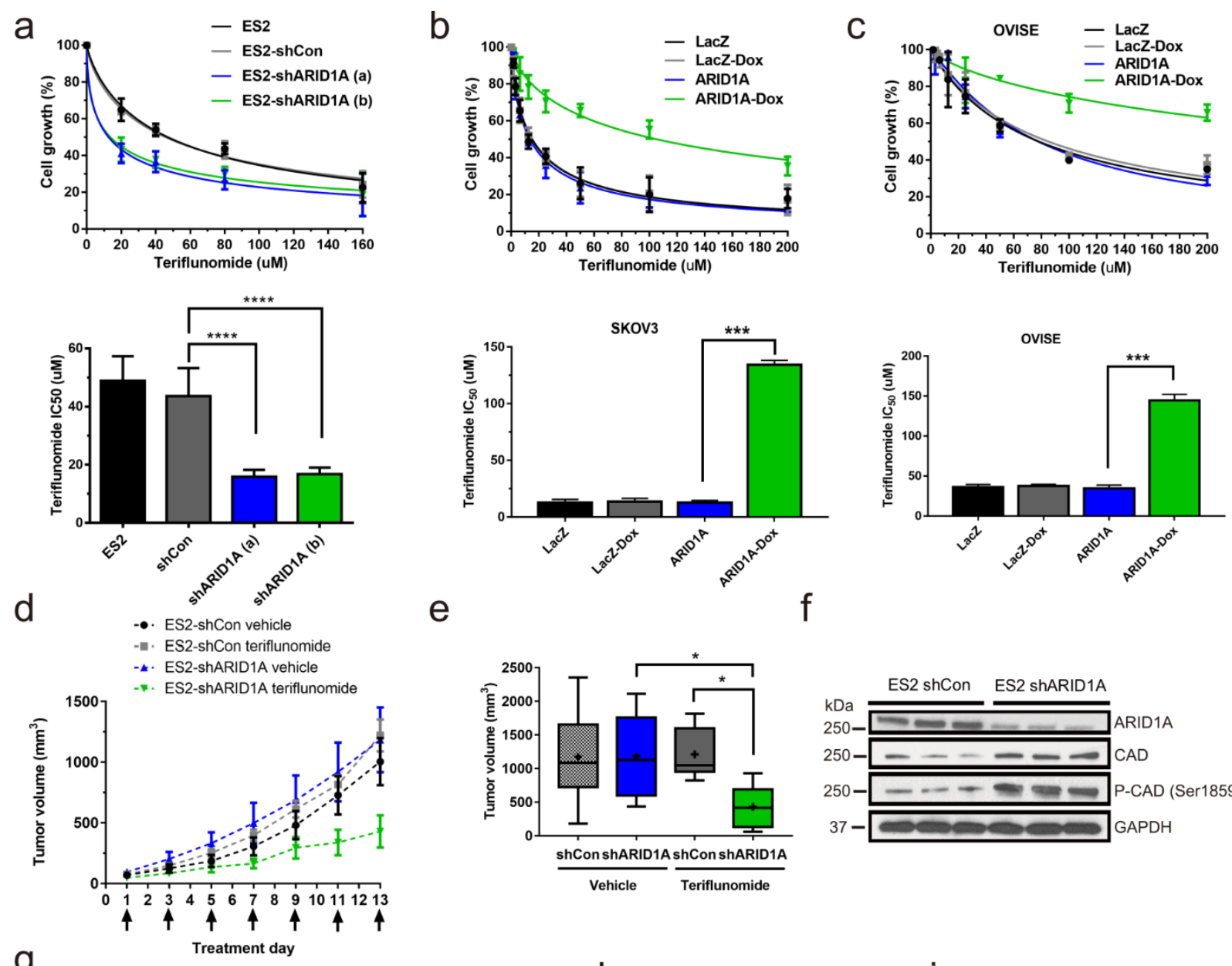

e
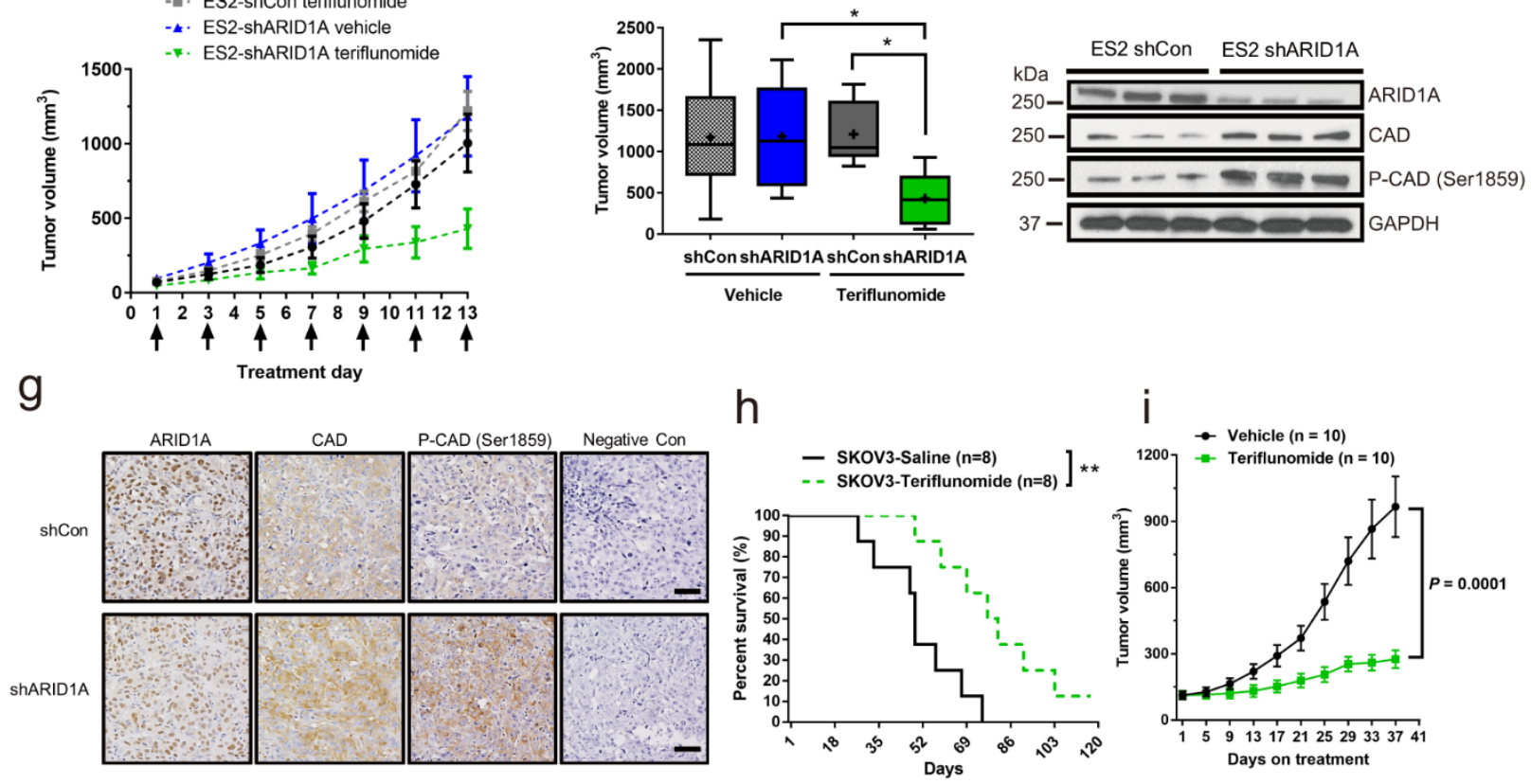

Figure 4. ARID1A-deficient cells and tumors display sensitization to de novo pyrimidine synthesis blockade therapy.

a, In the upper panel, the effect of teriflunomide in ES2 cells is quantified by showing the relative cell number following drug treatment at various concentrations for $72 \mathrm{~h}$. $A R I D 1 A-k n o c k d o w n$ cells, depicted by the blue line for shARID1A (a) and the green line for shARID1A (b), were more sensitive to teriflunomide, resulting in a decreased cell number following drug treatment, compared to untransfected cells (black line) or shCon cells (gray line). In the lower panel, the data shown in the upper panel was summarized by graphing the teriflunomide concentration that results in a $50 \%$ growth inhibitory effect $\left(\mathrm{IC}_{50}\right)$. The bars depict the mean $\mathrm{IC}_{50} \pm \mathrm{SD}$ for five independent experiments. 
Differences in $\mathrm{IC}_{50}$ were evaluated using one-way ANOVA with Tukey's post-test; ${ }^{* * *} P$ $<0.001$.

782

783

784

785

786

787

788

789

790

791

792

793

794

795

796

797

798

799

800

801

802

803

804

805

806

807

808

809

810

811

812

813

814

815

816

817

818

819

820

821

b, In the upper panel, the effect of teriflunomide is quantified in a single-clone SKOV3 cell line that was stably transfected with Lenti-puro-ARID1A-V5 (ARID1A) compared with control cells transfected with non-targeting Lenti-puro-LacZ-V5 (LacZ). ARID1Arestoration cells induced by doxycycline (Dox) (green line) were more resistant to teriflunomide, resulting in an increased cell number following drug treatment, compared to uninduced cells (blue line). In the lower panel, the data shown in the upper panel was summarized by graphing the $I_{50}$. The bars depict the mean $I_{50} \pm S D$ for three independent experiments. Differences in $\mathrm{IC}_{50}$ were evaluated using one-way ANOVA with Tukey's post-test; ${ }^{* * *} P<0.001$.

c, In the upper panel, the effect of teriflunomide is quantified in a single-clone OVISE cell line that was stably transfected with Lenti-puro-ARID1A-V5 (ARID1A) compared with control cells transfected with non-targeting Lenti-puro-LacZ-V5 (LacZ). ARID1Arestoration cells induced by Dox (green line) were more resistant to teriflunomide, resulting in an increased cell number following drug treatment, compared to uninduced cells (blue line). In the lower panel, the data shown in the upper panel was summarized by graphing the $\mathrm{IC}_{50}$. The bars depict the mean $\mathrm{IC}_{50} \pm \mathrm{SD}$ for three independent experiments. Differences in $\mathrm{IC}_{50}$ were evaluated using one-way ANOVA with Tukey's post-test; ${ }^{* \star *} P<0.001$.

d, The effect of teriflunomide was evaluated in vivo by treating xenograft-bearing mice every other day for 13 days (black arrows). The effect of teriflunomide on tumor xenograft growth is shown by depiction of the mean tumor volume $\pm S D(N=6$ to 8 animals/group) for each of the following groups: shCon treated with vehicle (black line), shARID1A treated with vehicle (blue line), shCon treated with teriflunomide (gray line), and shARID1A treated with teriflunomide (green line). Teriflunomide effectively inhibited tumor growth of shARID1A xenografts (green line), but not shCon xenografts (gray line).

e, The data shown in (d) are summarized by graphing the terminal tumor volumes. The bars depict the median (middle line) and mean (+) tumor volumes for each group. Differences between groups were evaluated using one-way ANOVA with Tukey's posttest; ${ }^{*} P<0.05$.

f, ARID1A, CAD, and phospho-CAD Ser1859 protein expression was evaluated in representative tumor xenograft cell lysates by immunoblotting. ARID1A-knockdown xenografts (shARID1A) showed decreased ARID1A protein levels and increased CAD and phospho-CAD protein levels. Equal protein loading was confirmed by Ponceau staining of the nitrocellulose membrane (not shown) and immunoblotting with an antiGAPDH antibody.

g, Representative immunohistology staining of xenograft tumor tissue sections for ARID1A, CAD, and phosphorylated CAD Ser1859 is shown. The negative-control samples underwent the same immunohistology staining procedure but without primary antibody incubation. Similar upregulation of CAD and P-CAD Ser1859 was observed in the ARID1A-knockdown xenograft tumor samples. Scale bar, $50 \mu \mathrm{m}$. 
$822 \mathbf{h}$, The effect of teriflunomide was evaluated in an ARID1A-deficient SKOV3 tumor

823 xenograft model. The xenograft model was generated by subcutaneously injecting

824 SKOV3 cells in Matrigel (1:1) into athymic nude mice. Teriflunomide (4 mg/kg) or

825 vehicle was intraperitoneally injected every other day. Tumor size was recorded on the

826 same day. A survival curve is shown; the terminal tumor volume is $1000 \mathrm{~mm}^{3}$.

827 Teriflunomide improved the survival of the tumor-bearing mice. The $P$ value was

828 calculated via two-tailed $t$-test. ${ }^{* *} P<0.01$. The effect of teriflunomide on an ES2-shCon

829 tumor xenograft model is shown in Figure S6.

830 i, PDXs (CTG-2213; ARID1A truncating mutation at GIn211*) were randomized into

831 vehicle control and Teriflunomide ( $4 \mathrm{mg} / \mathrm{kg}$ every other day). Tumor volume was

832 measured every four days. There was a significant decrease in treatment group relative

833 to control $(P=0.0001)$. The effect of teriflunomide on animal weight and individual

834 tumor growth is shown in Supplementary Fig. S7.

835

836

837

838

839

840

841

842

843

844

845

846

847

848

849

850

851 
bioRxiv preprint doi: https://doi.org/10.1101/2020.10.12.331975; this version posted October 12, 2020. The copyright holder for this preprint (which was not certified by peer review) is the author/funder, who has granted bioRxiv a license to display the preprint in perpetuity. It is made available under aCC-BY-NC-ND 4.0 International license.

a

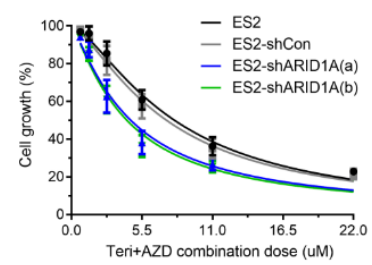

e

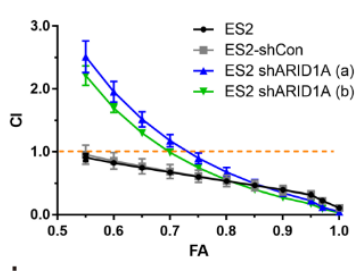

b

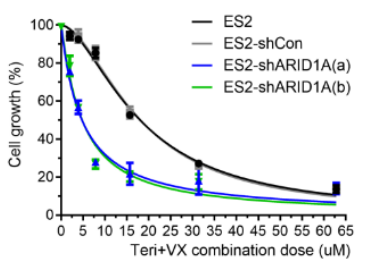

f

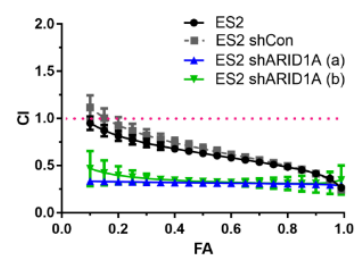

C

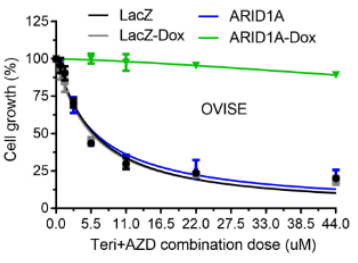

g

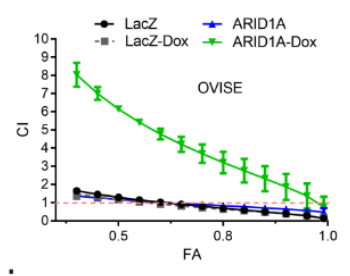

d

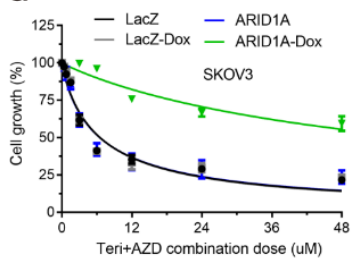

h

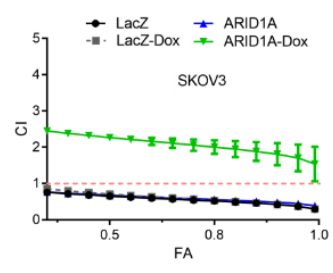

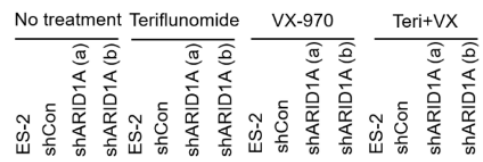

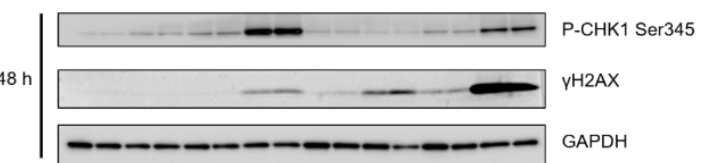

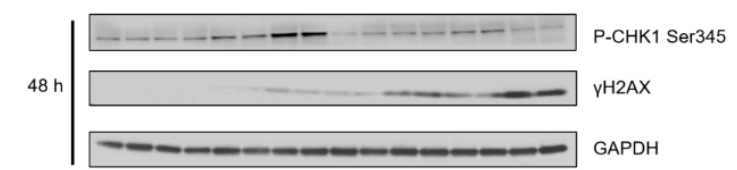

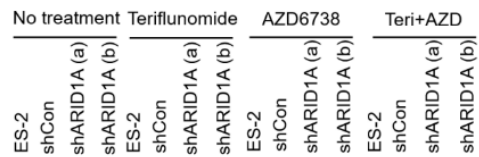

k
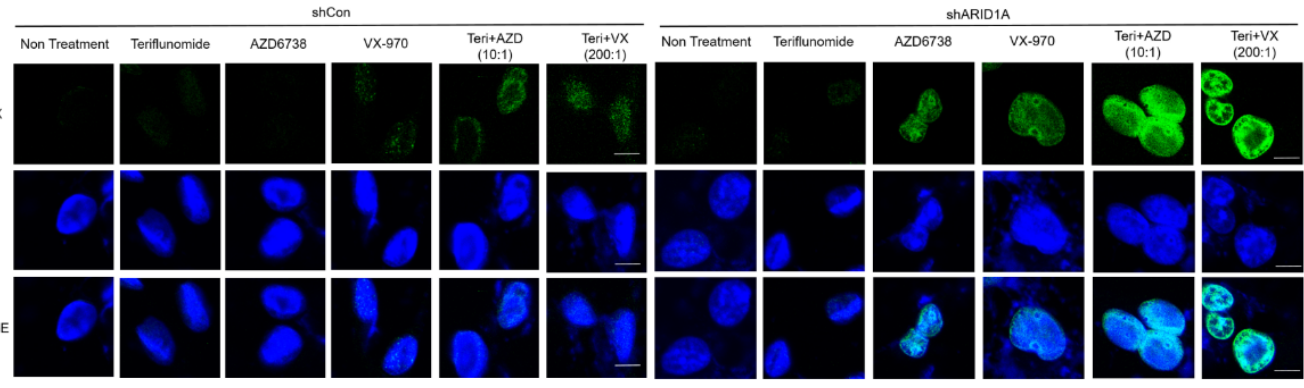

I
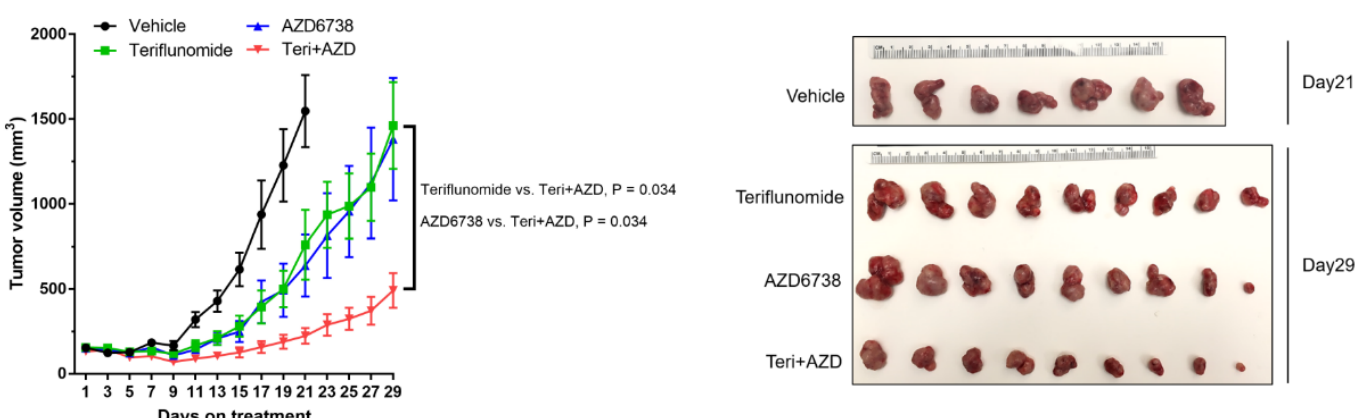

m
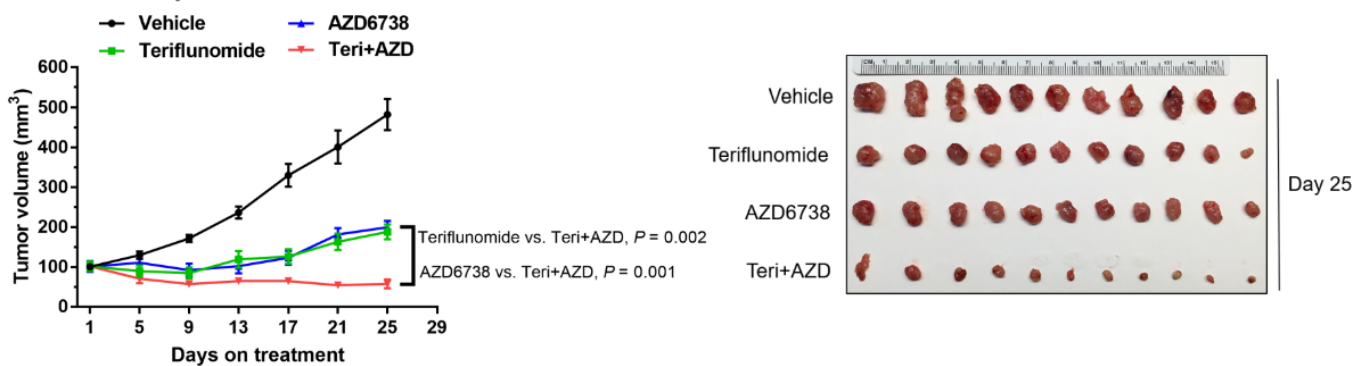


\section{Figure 5. Combination treatment with DHODHi and ATRi shows a synergistic} effect in ARID1A-deficient cells.

a, The effect of combination treatment of ES2 cells with teriflunomide and AZD6738 for $72 \mathrm{~h}$ is quantified. ARID1A-knockdown cells, depicted by the blue line for shARID1A (a) and the green line for shARID1A (b), were even more sensitive to combination treatment compared to untransfected cells (black line) or shCon cells (gray line). Representative images of cell morphology at $72 \mathrm{~h}$ are shown in Supplementary Figure S8.

b, The effect of combination treatment of ES2 cells with teriflunomide and VX-970 for 72 $\mathrm{h}$ is quantified. ARID1A-knockdown cells, as depicted by the blue line for shARID1A (a) and the green line for shARID1A (b), are even more sensitive to combination treatment compared to untransfected cells (black line) or shCon cells (gray line).

c, The effect of combination treatment of ARID1A-restoration OVISE cells with teriflunomide and AZD6738 for $72 \mathrm{~h}$ is quantified. ARID1A-induced cells (ARID1A-Dox), depicted by the green line, were even more resistant to combination treatment compared to non-ARID1A-induced cells.

d, The effect of combination treatment of ARID1A-restoration SKOV3 cells with teriflunomide and AZD6738 for $72 \mathrm{~h}$ is quantified. ARID1A-induced cells (ARID1A-Dox), depicted by the green line, were even more resistant to combination treatment compared to non-ARID1A-induced cells.

$\mathbf{e}-\mathbf{h}$, The data shown in (a-c) are summarized by graphing $\mathrm{FA}-\mathrm{Cl}$ plots, with $\mathrm{x}=$ fraction affected (FA) vs. $y=$ combination index $(\mathrm{Cl})$ (the Chou-Talalay plot). The $\mathrm{FA}-\mathrm{Cl}$ curves showed the detail synergic effect at each drug concentration. $\mathrm{Cl}<1,=1$, and $>1$ indicate synergism, an additive effect, and antagonism, respectively. Detailed analyses of the combination treatments in (a), (b), (c) and (d) are shown in Supplementary Tables S1, S5, S6 and S7, respectively.

i, $\mathrm{y}-\mathrm{H} 2 \mathrm{AX}$ protein expression was evaluated in ES2 cell lysates by immunoblotting at both $48 \mathrm{~h}$ and $72 \mathrm{~h}$. Teriflunomide $(15 \mu \mathrm{M})$ and AZD6738 $(1.5 \mu \mathrm{M})$ were used in singledrug and combination treatment groups. The target effect of AZD6738 on P-CHK1 Ser345 was decreased. ARID1A-knockdown cells (shARID1A) in the single-drug treatment groups showed increased $\mathrm{y}-\mathrm{H} 2 \mathrm{AX}$ protein levels, but the highest levels were in the Teri+AZD combination treatment group. Equal protein loading was confirmed by immunoblotting with an anti-GAPDH antibody.

j, The Teriflunomide+VX-970 combination treatment effect was evaluated by detecting $\mathrm{Y}-\mathrm{H} 2 \mathrm{AX}$ protein expression in ES2 cell lysates by immunoblotting at both $24 \mathrm{~h}$ and $48 \mathrm{~h}$. The target effect of VX-970 on P-CHK1 Ser345 was decreased. Teriflunomide $(15 \mu \mathrm{M})$ and VX-970 $(0.075 \mu \mathrm{M})$ were used in single-drug and combination treatment groups. ARID1A-knockdown cells (shARID1A) showed increased $\mathrm{y}-\mathrm{H} 2 \mathrm{AX}$ protein levels in the single-drug treatment groups, but the highest levels were in the combination treatment group. Equal protein loading was confirmed by immunoblotting with an anti-GAPDH antibody.

$\mathbf{k}$, Representative immunofluorescence staining of ES2 cells for $\mathrm{y}-\mathrm{H} 2 \mathrm{AX}$ (green) and

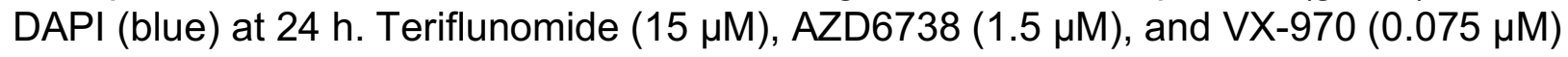


9

1

were used in single-drug and combination treatment groups. ARID1A-knockdown cells (shARID1A) showed increased $\mathrm{y}-\mathrm{H} 2 \mathrm{AX}$ protein levels following single-drug treatment groups, but the highest levels were in the combination treatment group. Scale bar, 10 $\mu \mathrm{m}$.

I, The effect of combination treatment with teriflunomide and AZD6738 was evaluated in vivo by treating ES2-shARID1A xenograft-bearing mice. The effect on tumor xenograft growth is shown by depiction of the mean tumor volume \pm s.e.m. $(\mathrm{N}=7$ to 9 animals/group) for each of the following groups: vehicle (black line, $N=7$ ), teriflunomide (green line, N=9), AZD6738 (blue line, N=9), and teriflunomide and AZD6738 (red line, $\mathrm{N}=9)$. Teriflunomide $(4 \mathrm{mg} / \mathrm{kg})$ or vehicle was intraperitoneally injected every other day. AZD6738 $(25 \mathrm{mg} / \mathrm{kg})$ or vehicle was given by oral gavage every day. Combination treatment more effectively inhibited tumor growth of shARID1A xenografts (red line) compared to single-drug-treated xenografts (green and blue lines). Images of all ES2shARID1A xenografts treated with vehicle control, teriflunomide, AZD6738, or teriflunomide plus AZD6738 is shown on the right side. The images are from the endpoint of scheduled treatment. The vehicle group reached maximum tumor on day 21. Tumor size on day 29 showed that Combination treatment more effectively inhibited tumor growth of shARID1A xenografts compared to single-drug-treated xenografts. Analysis of the correlation of tumor weight with tumor volume in each group is shown in Supplementary Figure S11.

$\mathbf{m}$, The effect of combination treatment with teriflunomide and AZD6738 was evaluated in PDXs (CTG-2213; ARID1A truncating mutation at GIn211*). The effect on tumor xenograft growth is shown by depiction of the mean tumor volume \pm s.e.m. $(\mathrm{N}=11$ animals/group) for each of the following groups: vehicle (black line), teriflunomide (green line), AZD6738 (blue line), and teriflunomide plus AZD6738 (red line). Combination treatment more effectively inhibited tumor growth (red line) compared to single-drug-treated xenografts (green and blue lines). The images of all PDXs treated with vehicle control, teriflunomide, AZD6738, or teriflunomide plus AZD6738 on the right side. The images are from the endpoint of scheduled treatment on day 25 . The combination treatment more effectively inhibited tumor growth of shARID1A xenografts compared to single-drug-treated xenografts. Analysis of the correlation of tumor weight with tumor volume in each group is shown in Supplementary Figure S11. 
937

938

939

940

941

942

943

944

945

946

947

948

949

950

951

952

953

954

955

956

957

958

959

960

961

962

963

964

965

966

967

\section{REFERENCES}

1 Wiegand, K. C. et al. ARID1A mutations in endometriosis-associated ovarian carcinomas. New England Journal of Medicine 363, 1532-1543 (2010).

2 Jones, S. et al. Frequent mutations of chromatin remodeling gene ARID1A in ovarian clear cell carcinoma. Science 330, 228-231 (2010).

3 Wu, R.-C., Wang, T.-L. \& Shih, I.-M. The emerging roles of ARID1A in tumor suppression. Cancer biology \& therapy 15, 655-664 (2014).

4 Maeda, D. et al. Clinicopathological significance of loss of ARID1A immunoreactivity in ovarian clear cell carcinoma. International journal of molecular sciences 11, 5120-5128 (2010).

5 Wilson, B. G. \& Roberts, C. W. SWI/SNF nucleosome remodellers and cancer. Nature Reviews Cancer 11, 481 (2011).

$6 \mathrm{Li}$, J. et al. Epigenetic driver mutations in ARID1A shape cancer immune phenotype and immunotherapy. The Journal of Clinical Investigation (2020).

7 Katagiri, A. et al. Loss of ARID1A expression is related to shorter progression-free survival and chemoresistance in ovarian clear cell carcinoma. Modern Pathology 25, 282-288 (2012).

8 Forbes, S. A. et al. COSMIC: somatic cancer genetics at high-resolution. Nucleic Acids Research 45, D777-D783 (2016).

9 Anglesio, M. S. et al. Type-specific cell line models for type-specific ovarian cancer research. PloS one 8, e72162 (2013).

10 Dykhuizen, E. C. et al. BAF complexes facilitate decatenation of DNA by topoisomerase Ila. Nature 497, 624 (2013).

11 Guan, B., Wang, T.-L. \& Shih, I.-M. ARID1A, a factor that promotes formation of SWI/SNF-mediated chromatin remodeling, is a tumor suppressor in gynecologic cancers. Cancer research 71, 6718-6727 (2011).

12 Ben-Sahra, I., Howell, J. J., Asara, J. M. \& Manning, B. D. Stimulation of de novo pyrimidine synthesis by growth signaling through mTOR and S6K1. Science 339, 1323-1328 (2013).

13 Robitaille, A. M. et al. Quantitative phosphoproteomics reveal mTORC1 activates de novo pyrimidine synthesis. Science 339, 1320-1323 (2013). 
968

969

970

971

972

973

974

975

976

977

978

979

980

981

982

983

984

985

986

987

988

989

990

991

992

993

994

995

996

997

14 Barretina, J. et al. The Cancer Cell Line Encyclopedia enables predictive modelling of anticancer drug sensitivity. Nature 483, 603 (2012).

15 Ruiz-Ramos, A., Velázquez-Campoy, A., Grande-García, A., Moreno-Morcillo, M. \& Ramón-Maiques, S. Structure and functional characterization of human aspartate transcarbamoylase, the target of the anti-tumoral drug PALA. Structure 24, 10811094 (2016).

16 O'Connor, P. et al. Randomized trial of oral teriflunomide for relapsing multiple sclerosis. New England Journal of Medicine 365, 1293-1303 (2011).

17 Williamson, C. T. et al. ATR inhibitors as a synthetic lethal therapy for tumours deficient in ARID1A. Nature Communications 7 (2016).

18 Chou, T.-C. Drug combination studies and their synergy quantification using the Chou-Talalay method. Cancer research 70, 440-446 (2010).

19 Wild, J., Loughrey-Chen, S. \& Corder, T. In the presence of CTP, UTP becomes an allosteric inhibitor of aspartate transcarbamoylase. Proceedings of the National Academy of Sciences 86, 46-50 (1989).

20 Rabinowitz, J. D. et al. Dissecting enzyme regulation by multiple allosteric effectors: nucleotide regulation of aspartate transcarbamoylase. Biochemistry 47, 5881-5888 (2008).

21 Rees, S. et al. Bicistronic vector for the creation of stable mammalian cell lines that predisposes all antibiotic-resistant cells to express recombinant protein.

Biotechniques 20, 102-104, 106, 108-110 (1996).

22 Han, Y. et al. Proteomic investigation of the interactome of FMNL1 in hematopoietic cells unveils a role in calcium-dependent membrane plasticity. Journal of proteomics 78, 72-82 (2013).

23 Yang, C.-P. H. \& Horwitz, S. B. Taxol mediates serine phosphorylation of the 66kDa Shc isoform. Cancer Research 60, 5171-5178 (2000).

24 Keller, D. M., Zeng, S. X. \& Lu, H. in p53 Protocols. 121-133 (Springer, Totowa, NJ, 2003).

25 Ran, F. A. et al. Genome engineering using the CRISPR-Cas9 system. Nature protocols 8, 2281 (2013). 
99826 Huang, G. S. et al. Insulin-like growth factor 2 expression modulates Taxol

999 resistance and is a candidate biomarker for reduced disease-free survival in ovarian

1000 cancer. Clinical Cancer Research 16, 2999-3010 (2010).

100127 Reagan-Shaw, S., Nihal, M. \& Ahmad, N. Dose translation from animal to human

1002 studies revisited. The FASEB Journal 22, 659-661 (2008).

1003 